Canad. J. Math. Vol. 71 (5), 2019 pp. 1061-1101

http://dx.doi.org/10.4153/CJM-2018-030-8

(c) Canadian Mathematical Society 2019

\title{
A Basis Theorem for the Degenerate Affine Oriented Brauer-Clifford Supercategory
}

\author{
Jonathan Brundan, Jonathan Comes, and Jonathan Robert Kujawa
}

\begin{abstract}
We introduce the oriented Brauer-Clifford and degenerate affine oriented Brauer-Clifford supercategories. These are diagrammatically defined monoidal supercategories that provide combinatorial models for certain natural monoidal supercategories of supermodules and endosuperfunctors, respectively, for the Lie superalgebras of type Q. Our main results are basis theorems for these diagram supercategories. We also discuss connections and applications to the representation theory of the Lie superalgebra of type Q.
\end{abstract}

\section{Introduction}

\subsection{Overview}

Let $\mathbb{k}$ denote a fixed ground field ${ }^{1}$ of characteristic not two. In this paper we study certain monoidal supercategories, that is, categories in which morphisms form $\mathbb{Z}_{2}$-graded $\mathbb{k}$-vector spaces, the category has a tensor product, and compositions and tensor products of morphisms are related by a graded version of the interchange law (see Section 2 for more details). While enriched monoidal categories have been the object of study for some time, it is only recently that monoidal supercategories have taken on a newfound importance thanks to the role they play in higher representation theory. To name a few examples, they appear explicitly or implicitly in the categorification of Heisenberg algebras [RS], "odd" categorifications of Kac-Moody (super)algebras (e.g., [EL, KKO1, KKO2]), the definition of super Kac-Moody categories [BE2], and in various Schur-Weyl dualities in the $\mathbb{Z}_{2}$-graded setting (e.g., [KT]).

In this paper we introduce two monoidal supercategories. They are the oriented Brauer-Clifford supercategory $(\mathrm{OBC})$ and the degenerate affine oriented BrauerClifford supercategory $(\mathcal{A O B C})$. They are defined by generators and relations. For both monoidal supercategories the generating objects are $\uparrow$ and $\downarrow$. Hence, objects in both $\mathcal{O B C}$ and $\mathcal{A O B C}$ can be viewed as finite words in $\uparrow$ and $\downarrow$ (we write $\mathbb{1}$ for the unit object). For $\mathcal{O B C}$ the generating morphisms are the three even morphisms $\mathcal{U}: \mathbb{1} \rightarrow \uparrow \downarrow, \curvearrowleft: \downarrow \uparrow \rightarrow \mathbb{1}, \widehat{\chi}: \uparrow \uparrow \rightarrow \uparrow \uparrow$, and one odd morphism $\hat{\phi}: \uparrow \rightarrow \uparrow$. For $\mathcal{A O B C}$, the generating morphisms are those of $\mathcal{O B C}$ along with an even morphism $\hat{\phi}: \uparrow \rightarrow \uparrow$. These generating morphisms are subject to an explicit list of local relations (see Definitions 3.2 and 3.7 for details). In Section 3 we explain how more complicated diagrams can be interpreted as morphisms in $\mathcal{O B C}$ and $\mathcal{A O B} \mathcal{C}$. For example,

Received by the editors September 18, 2017; revised April 20, 2018.

Published electronically January 11, 2019.

AMS subject classification: 17B10, 18D10.

Keywords: monoidal category, supercategory, Lie superalgebra, type Q.

${ }^{1}$ More generally, one could take $\mathbb{k}$ to be a commutative ring. See Section 6.2. 
here are two diagrams that correspond to morphisms in $\operatorname{Hom}_{\mathcal{A O B C}}\left(\downarrow^{2} \uparrow^{2}, \downarrow^{2} \uparrow^{3} \downarrow\right)$ :
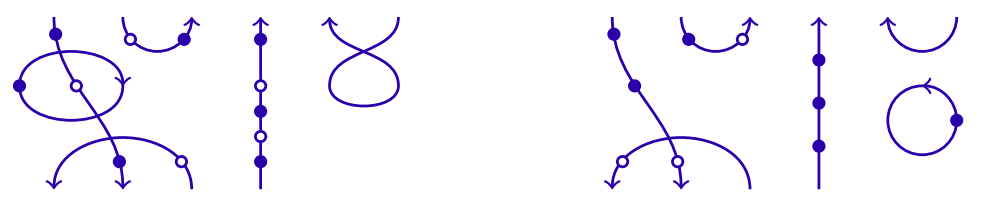

In particular, it will be obvious that the Hom-spaces in $\mathcal{O B C}$ and $\mathcal{A O B C}$ are spanned by the set of all such diagrams they contain. As is often the case, the difficulty is in identifying a subset of these diagrams that form a basis. The main results of this paper are contained in Theorems 3.4 and 3.9 in which we provide a diagrammatic basis for the morphism spaces of these supercategories, and Theorem 7.1 in which we provide bases for the cyclotomic quotients of $\mathcal{A O B}$.

\subsection{Motivation and Applications}

Let us describe the motivation for these supercategories and some consequences of the aforementioned basis theorems. Let

$$
\mathfrak{q}=\mathfrak{q}(n)=\left\{\left(\begin{array}{ll}
A & B \\
B & A
\end{array}\right) \mid A, B \text { are } n \times n \text { matrices with entries in } \mathbb{k}\right\} .
$$

Put a $\mathbb{Z}_{2}$-grading on $\mathfrak{q}=\mathfrak{q}_{\overline{0}} \oplus \mathfrak{q}_{\overline{1}}$ by setting $\mathfrak{q}_{\overline{0}}$ (resp. $\mathfrak{q}_{\overline{1}}$ ) to be the subspace of matrices with $B=0$ (resp. $A=0$ ). Then $\mathfrak{q}$ is the Lie superalgebra of type $Q$, where the Lie bracket given by the graded version of the commutator bracket. See Section 4.1 for details.

The representations in type $Q$ do not have a classical analogue. Despite the important early work done by Penkov-Serganova, and others to obtain character formulas and other information (see [PS, Brul] and references therein), the representation theory in type $\mathrm{Q}$ remains mysterious. For example, only very recently the structure of category $\mathcal{O}$ for $\mathfrak{q}$ became clear thanks to the work of Chen [Che], Cheng, Kwon, and Wang [CKW], and Brundan and Davidson [BD2, BD3].

Since the enveloping superalgebra of $\mathfrak{q}, U(\mathfrak{q})$, is a Hopf superalgebra, one can consider the tensor product of $\mathfrak{q}$-supermodules and the duals of finite-dimensional $\mathfrak{q}$-supermodules. Let $V$ denote the natural supermodule for $\mathfrak{q}$, that is, column vectors of height $2 n$ with the action of $\mathfrak{q}$ given by matrix multiplication. Using the Hopf structure we can then form tensor products of $V$ and its dual, $V^{*}$. For brevity, let us write $V^{\uparrow}=V$ and $V^{\downarrow}=V^{*}$, and, more generally, given a word a in $\uparrow$ and $\downarrow$, let $V^{\text {a denote }}$ the tensor product of the corresponding supermodules (e.g., $V^{\uparrow \uparrow \downarrow}=V \otimes V \otimes V^{*}$ ). The full subsupercategory of all $\mathfrak{q}$-supermodules obtained in this way is a natural object of study.

Moreover, the translation superfunctors given by tensoring with $V$ 's and $V^{*}$ 's are a key tool in much of the progress made in the study of type Q representations. That is, an important role is played by the full subsupercategory of endosuperfunctors of the form $V^{\mathrm{a}} \otimes-$ as a ranges over all finite words in $\uparrow$ and $\downarrow$. Given the importance of these endosuperfunctors, it is of interest to understand this supercategory. 
By design, $\mathcal{O B C}$ and $\mathcal{A O B C}$, respectively, are combinatorial models for these two supercategories. Specifically, there is a monoidal superfunctor

$$
\Phi: \mathcal{O B C} \longrightarrow \mathfrak{q} \text {-supermodules }
$$

given on objects by $\Phi(\mathrm{a})=V^{\mathrm{a}}$. When $\mathbb{k}$ is characteristic zero, this superfunctor is full (see Theorem 4.1). That is, there is a surjective superalgebra homomorphism

$$
\operatorname{End}_{\mathcal{O B C}}(\mathrm{a}) \longrightarrow \operatorname{End}_{\mathfrak{q}(n)}\left(V^{\mathrm{a}}\right)
$$

which, moreover, is an isomorphism whenever the length of a is less than or equal to $n$ (see Remark 4.2).

It follows from our basis theorem that $\operatorname{End}_{\mathcal{O B C}}\left(\uparrow^{r}\right)$ is isomorphic to the (finite) Sergeev superalgebra introduced in [Ser2] (see Corollary 3.5). For arbitrary a, $\operatorname{End}_{\mathcal{O B C}}(\mathrm{a})$ is isomorphic to the walled Brauer-Clifford superalgebra introduced by Jung and Kang [JK] (see Corollary 3.6). The fact that Section 1.2 is an isomorphism whenever the length of a is less than or equal to $n$ recovers [JK, Theorem 3.5]. We should point out the definitions given in [JK] are global in nature. For example, it is not a priori clear their intricate rule defines an associative product. In contrast, our diagrammatic description for these superalgebras involves only local relations and leads to significant simplifications.

Analogously, there is a monoidal superfunctor from $\mathcal{A O B C}$ to the supercategory of endosuperfunctors of $\mathfrak{q}$-supermodules, $\Psi: \mathcal{A O B C} \rightarrow \mathcal{E} n d(U(\mathfrak{q})$-smod), given on objects by $\Psi(\mathrm{a})=V^{\mathrm{a}} \otimes-$. When $\mathbb{k}$ is characteristic zero, this superfunctor is faithful "asymptotically" in the sense that given any nonzero morphism in $\mathcal{A O B C}$, its image under $\Psi$ is nonzero as long as $n$ is sufficiently large. Indeed, this observation is key to proving the basis theorems. We reduce to showing that the induced map

$$
\operatorname{End}_{\mathcal{A O B C}}\left(\uparrow^{r}\right) \longrightarrow \operatorname{End}_{\mathcal{E} n d(U(\mathfrak{q}) \text {-smod })}\left(V^{\otimes r} \otimes-\right)
$$

is injective for $n$ sufficiently large (e.g., $n \geq r)$. This in turn is proven by introducing a certain $\mathfrak{q}(n)$-supermodule $M$ (which we call the generic Verma supermodule) and proving that the induced map of superalgebras

$$
\operatorname{End}_{\mathcal{A O B} \mathcal{B}}\left(\uparrow^{r}\right) \longrightarrow \operatorname{End}_{\mathfrak{q}(n)}\left(V^{\otimes r} \otimes M\right)
$$

is injective.

As an application, in Section 4.4 we use $\Psi$ to compute a family of central elements in $U(\mathfrak{q})$. By the basis theorem $\operatorname{End}_{\mathcal{A O B C}}(\mathbb{1})$ is known to be a polynomial ring in $\Delta_{1}, \Delta_{3}, \Delta_{5}, \ldots$, where $\Delta_{k}$ is defined by Section 3.5. In Section 4.4, we explicitly compute the central element corresponding to $\Psi\left(\Delta_{k}\right)$ for each $k$ and show that they are essentially the central elements first introduced by Sergeev [Ser1] after the application of the antipode of $U(\mathfrak{q})$ (see Proposition 4.6).

\subsection{Cyclotomic Quotients}

Fix nonnegative integers $a, b$, and $m_{i} \in \mathbb{k}$ for each $1 \leq i \leq a$. Using this data, fix the polynomial of degree $\ell:=2 a+b$ given by $f(t)=t^{b} \prod_{1 \leq i \leq a}\left(t^{2}-m_{i}\right)$. The cyclotomic quotient $\mathcal{O B C}^{f}$ is the supercategory defined as the quotient of $\mathcal{A O B C}$ by the left tensor ideal generated by $f(\hat{\phi})$. Note that $\mathcal{O B C}^{f}$ does not obviously inherit the structure 
of a monoidal supercategory from $\mathcal{A O B C}$, but it is a left module supercategory over $\mathcal{A O B C}$. In Section 7, we describe a basis for the Hom-spaces of $\mathcal{O B C}^{f}$ (see Theorem 7.1). That this forms a basis was conjectured in a preprint version of this article (written by the second two authors). The first author provided a proof soon after the preprint appeared on the ArXiv; see Section 8. Subsequently, Gao, Rui, Song, and Su posted an independent proof of Theorem 7.1 in [GRSS2]; their proof is in the spirit of our proof of Theorem 3.9.

\subsection{Connection to the Superalgebras of Gao, Rui, Song, and Su}

Shortly after the authors released the first version of this paper, Gao-Rui-Song-Su posted a preprint to the ArXiv in which they describe their independent work on affine walled Brauer-Clifford superalgebras and their cyclotomic quotients [GRSS1]. In the same spirit as this paper, they define these superalgebras by generators and relations and provide basis theorems. The key difference is that we choose to work with the supercategories, whereas they work with superalgebras. In Section 7.2 we explain the connection between these two approaches and show their superalgebras are specializations of endomorphism superalgebras in our supercategories. To do so, we construct explicit superalgebra maps from the Gao-Rui-Song-Su superalgebras to the endomorphism superalgebras of our supercategories. Using our bases theorems, one can check that the images under those superalgebra maps of the so-called regular monomials in the superalgebras of Gao, Rui, Song, and Su are bases of the appropriate endomorphism superalgebras. Whence, our basis theorems imply the linear independence of the corresponding basis theorems of Gao, Rui, Song, and Su ( [GRSS1, Theorems 5.15 and 6.10]). These arguments cannot merely be reversed in order to obtain our basis theorems from those in [GRSS1]. The main obstacle comes from the fact that our endomorphism superalgebras do not come equipped with nice descriptions via generators and relations. Indeed, without already having a basis theorem for the supercategory, it is difficult to extract a full system of generators and relations for the endomorphism superalgebras from the defining generators and relations for the monoidal supercategory. Hence, providing a well-defined superalgebra map from our endomorphism superalgebras to the superalgebras of Gao, Rui, Song, and Su is not an easy task. In particular, we are unable to conclude Theorem 6.3 from [GRSS1, Theorem 5.14] nor Theorem 7.1 from [GRSS1, Theorem 6.10].

\subsection{Future Directions}

There are a number of intereting questions yet to be considered. For example, in his $\mathrm{PhD}$ thesis, Reynolds showed that the locally finite-dimensional representations of a certain specialization of the oriented Brauer category provide a categorification of the tensor product of a highest weight representation and lowest weight representation for a Kac-Moody Lie algebra of type A [Rey]. See [Bru3] for the quantum version of this story. We expect similar results to hold for $O \mathcal{O C}$ where the categorification is of a representation for a Kac-Moody algebra of type B. Another natural question is to extend the results of this paper from the classical to the quantum setting. There is a quantized enveloping superalgebra, $U_{q}(\mathfrak{q})$, which is a Hopf superalgebra and one can ask 
for quantum analogues of $\mathcal{O B C}$ and $\mathcal{A O B C}$. The quantum walled Brauer-Clifford superalgebras were already introduced in $[\mathrm{BGH}+]$. In a third direction, there should be representations of $\mathcal{A O B C}$ and its cyclotomic quotients related to the representations of finite $W$-superalgebras of type Q and to an expected higher level mixed SchurWeyl-Sergeev duality (see [BCNR, Section 4.6] where this is explained for type A). So far as the authors are aware, this theory has yet to be developed.

\section{Monoidal Supercategories}

In this section we give a brief introduction to monoidal supercategories following $[\mathrm{BE} 1, \$ 1]$. We refer the reader to $l o c$. cit. for more details and further references.

\subsection{Superspaces}

Let $\mathbb{k}$ be a fixed ground field of characteristic not two. A superspace $V=V_{\overline{0}} \oplus V_{\overline{1}}$ is a $\mathbb{Z}_{2^{-}}$ graded $\mathbb{k}$-vector space. As we will also have $\mathbb{Z}$-gradings, we reserve the word degree for later use and instead refer to the parity of an element. That is, elements of $V_{\overline{0}}$ (resp. $V_{\overline{1}}$ ) are said to have parity $\overline{0}$ or to be even (resp. parity $\overline{1}$ or $o d d$ ). Given a homogeneous element $v \in V$, we write $|v| \in \mathbb{Z}_{2}$ for the parity of the element. Given two superspaces $V$ and $W$, the set of all linear maps $\operatorname{Hom}_{\mathbb{k}}(V, W)$ is naturally $\mathbb{Z}_{2}$-graded by declaring that $f: V \rightarrow W$ has parity $\varepsilon \in \mathbb{Z}_{2}$ if $f\left(V_{\mathcal{\varepsilon}^{\prime}}\right) \subseteq V_{\varepsilon+\varepsilon^{\prime}}$ for all $\varepsilon^{\prime} \in \mathbb{Z}_{2}$. Let $\mathfrak{s v e c}$ and $\underline{\mathfrak{s v e c}}$ denote the categories of all superspaces with $\operatorname{Hom}_{\mathfrak{s v e c}}(V, W)=\operatorname{Hom}_{\mathbb{k}}(V, W)$ and $\operatorname{Hom}_{\mathfrak{s \mathfrak { v e c }}}(V, W)=\operatorname{Hom}_{\mathbb{k}}(V, W)_{\overline{0}}$.

Given superspaces $V$ and $W$, the vector space tensor product $V \otimes W$ is also naturally a superspace with $\mathbb{Z}_{2}$-grading given by declaring $|v \otimes w|=|v|+|w|$ for all homogeneous $v \in V$ and $w \in W$. The tensor product of linear maps between superspaces is defined via $(f \otimes g)(v \otimes w)=(-1)^{|g| v \mid} f(v) \otimes g(w)$. This gives $\underline{\mathfrak{s w e c}}$ (but not $\mathfrak{s v e c}$ ) the structure of a monoidal category with $\mathbb{1}=\mathbb{k}$ (viewed as superspace concentrated in even parity). The graded flip map $v \otimes w \mapsto(-1)^{|v||w|} w \otimes v$ gives $\mathfrak{s v e c}$ the structure of a symmetric monoidal category. Here and elsewhere we write the formula only for homogeneous elements with the general case given by extending linearly.

\subsection{Monoidal Supercategories}

By a supercategory we mean a category enriched in $\underline{\mathfrak{s v e c}}$. Similarly, a superfunctor is a functor enriched in $\mathfrak{s w e c}$. Given two superfunctors $F, G: \mathcal{A} \rightarrow \mathcal{B}$, a supernatural transformation $\eta: F \rightarrow G$ consists of $\eta_{\mathrm{a}, \varepsilon} \in \operatorname{Hom}_{\mathcal{B}}(F a, G a)_{\varepsilon}$ for each object a $\in \mathcal{A}$ and $\varepsilon \in \mathbb{Z}_{2}$ such that

$$
\eta_{\mathrm{b}, \varepsilon} \circ F f=(-1)^{\varepsilon|f|} G f \circ \eta_{\mathrm{a}, \varepsilon}
$$

for every $f \in \operatorname{Hom}_{\mathcal{A}}(\mathrm{a}, \mathrm{b})$. We will write $\eta_{\mathrm{a}}=\eta_{\mathrm{a}, \overline{0}}+\eta_{\mathrm{a}, \overline{\mathrm{l}}} \in \operatorname{Hom}_{\mathcal{B}}(F \mathrm{a}, \mathrm{Ga})$. The space of all supernatural transformations from $F$ to $G$ is given the structure of a superspace by declaring a supernatural transformation $\eta$ to be even (resp. odd) if $\eta_{\mathrm{a}, \overline{\mathrm{l}}}=0$ (resp. $\eta_{\mathrm{a}, \overline{0}}=0$ ) for all objects a.

Given supercategories $\mathcal{A}$ and $\mathcal{B}$, there is a supercategory $\mathcal{A} \otimes \mathcal{B}$ whose objects are pairs ( $a, b)$ of objects $a \in \mathcal{A}$ and $b \in \mathcal{B}$ and whose morphisms are given by the tensor 
product of superspaces

$$
\operatorname{Hom}_{\mathcal{A} \otimes \mathcal{B}}\left((a, b),\left(a^{\prime}, b^{\prime}\right)\right)=\operatorname{Hom}_{\mathcal{A}}\left(a, a^{\prime}\right) \otimes \operatorname{Hom}_{\mathcal{B}}\left(b, b^{\prime}\right),
$$

with composition defined using the symmetric braiding on $\underline{\mathfrak{s v e c}}$ :

$$
(f \otimes g) \circ(h \otimes k)=(-1)^{|g| h \mid}(f \circ h) \otimes(g \circ k) .
$$

This can be used to give the category $\mathfrak{s c a t}$ of all supercategories the structure of a monoidal category.

By a monoidal supercategory we mean a supercategory $\mathcal{A}$ equipped with a superfunctor $-\otimes-: \mathcal{A} \otimes \mathcal{A} \rightarrow \mathcal{A}$, a unit object $\mathbb{1}$, and even supernatural isomorphisms

$$
(-\otimes-) \otimes-\stackrel{\sim}{\longrightarrow}-\otimes(-\otimes-) \text { and } \mathbb{1} \otimes-\stackrel{\sim}{\longrightarrow}-\stackrel{\sim}{\longleftarrow}-\otimes \mathbb{1}
$$

called coherence maps satisfying certain axioms analogous to the ones for a monoidal category. A monoidal supercategory is called strict if its coherence maps are identities. A monoidal superfunctor between two monoidal supercategories $\mathcal{A}$ and $\mathcal{B}$ is a superfunctor $F: \mathcal{A} \rightarrow \mathcal{B}$ equipped with an even supernatural isomorphism $(F-) \otimes(F-) \stackrel{\sim}{\longrightarrow} F(-\otimes-)$ and an even isomorphism $\mathbb{1}_{\mathcal{B}} \stackrel{\sim}{\longrightarrow} F \mathbb{1}_{\mathcal{A}}$ satisfying axioms analogous to the ones for a monoidal functor.

A braided monoidal supercategory is a monoidal supercategory $\mathcal{A}$ equipped with a $\mathfrak{s v e c}$-enriched version of a braiding. More precisely, let $T: \mathcal{A} \otimes \mathcal{A} \rightarrow \mathcal{A}$ denote the superfunctor defined on objects by $(\mathrm{a}, \mathrm{b}) \mapsto \mathrm{b} \otimes \mathrm{a}$ and on morphisms by $f \otimes g \mapsto$ $(-1)^{|f||g|} g \otimes f$. A braiding on a $\mathcal{A}$ is a supernatural isomorphism $\gamma:-\otimes-\rightarrow T$ satisfying the usual hexagon axioms. A symmetric monoidal supercategory is a braided monoidal supercategory $\mathcal{A}$ with $\gamma_{\mathrm{a}, \mathrm{b}}^{-1}=\gamma_{\mathrm{b}, \mathrm{a}}$ for all objects $\mathrm{a}, \mathrm{b} \in \mathcal{A}$.

Given a monoidal supercategory $\mathcal{A}$ and an object a $\in \mathcal{A}$, by a (left) dual to a we mean an object $a^{*}$ equipped with homogeneous evaluation and coevaluation morphisms $\mathrm{ev}_{\mathrm{a}}: \mathrm{a}^{*} \otimes \mathrm{a} \rightarrow \mathbb{1}$ and $\operatorname{coev}_{\mathrm{a}}: \mathbb{1} \rightarrow \mathrm{a} \otimes \mathrm{a}^{*}$, respectively, in which $\mathrm{ev}_{\mathrm{a}}$ and $\operatorname{coev}_{a}$ have the same parity and satisfy the super version of the usual adjunction axioms. For example, given a finite-dimensional superspace $V$ with homogeneous basis $\left\{v_{i} \mid i \in I\right\}, V^{*}=\operatorname{Hom}_{\mathbb{k}}(V, \mathbb{k})$ with evaluation and coevaluation given by $f \otimes v \mapsto f(v)$ and $1 \mapsto \sum_{i \in I} v_{i} \otimes v_{i}^{*}$ respectively, where $v_{i}^{*} \in V^{*}$ is defined by $v_{i}^{*}\left(v_{j}\right)=\delta_{i, j}$. A monoidal supercategory in which every object has a (left) dual is called (left) rigid.

The following examples will be relevant for what follows.

(i) Any $\mathbb{k}$-linear monoidal category can be viewed as a monoidal supercategory in which all Hom-spaces are concentrated in parity $\overline{0}$. If the category is braided, symmetric braided, or rigid, then it still is braided as a supercategory.

(ii) The tensor product and braiding defined in Section 2.1 give $\mathfrak{s v e c}$ the structure of a symmetric monoidal supercategory with $\mathbb{1}=\mathbb{k}$ (viewed as a superspace concentrated in parity $\overline{0}$ ). The symmetric braiding $\gamma_{V, W}: V \otimes W \rightarrow W \otimes V$ is given by the graded flip map. The full subsupercategory of finite-dimensional superspaces is rigid.

(iii) Given a Lie superalgebra $\mathfrak{g}=\mathfrak{g}_{0} \oplus \mathfrak{g}_{\overline{1}}$ over a field $\mathbb{k}$ of characteristic not two, let $\mathfrak{g}$-smod denote the supercategory of all $\mathfrak{g}$-supermodules. That is, superspaces $M=$ $M_{\overline{0}} \oplus M_{\overline{1}}$ with an action by $\mathfrak{g}$ that respects the grading in the sense that $\mathfrak{g}_{\varepsilon} \cdot M_{\mathcal{\varepsilon}^{\prime}} \subseteq$ $M_{\varepsilon+\varepsilon^{\prime}}$. The tensor product $M \otimes M^{\prime}$ has action given by $x .\left(m \otimes m^{\prime}\right)=(x . m) \otimes m^{\prime}+$ $(-1)^{|x||m|} m \otimes\left(x . m^{\prime}\right)$ for all homogeneous $x \in \mathfrak{g}, m \in M$, and $m^{\prime} \in M^{\prime}$ and the graded 
flip map provides a symmetric braiding. The unit object $\mathbb{1}$ is the ground field $\mathbb{k}$ with trivial $\mathfrak{g}$-action. In this way, $\mathfrak{g}$-smod is a symmetric monoidal supercategory. The full subsupercategory of finite-dimensional $\mathfrak{g}$-supermodules is rigid with the action given on $M^{*}$ by $(x . f)(m)=-(-1)^{|x||f|} f(x . m)$.

(iv) Given a supercategory $\mathcal{A}$, let $\mathcal{E} n d(\mathcal{A})$ denote the supercategory whose objects are all superfunctors $\mathcal{A} \rightarrow \mathcal{A}$ with supernatural transformations as morphisms. We give $\mathcal{E} n d(\mathcal{A})$ the structure of a monoidal supercategory with $\mathbb{1}=\mathrm{Id}$ as follows. The tensor product of two superfunctors $F, G: \mathcal{A} \rightarrow \mathcal{A}$ is defined by composition $F \otimes G=$ $F \circ G$. Given supernatural transformations $\eta: F \rightarrow G$ and $\theta: H \rightarrow K$, we define $\eta \otimes \theta: F \circ H \rightarrow G \circ K$ by setting $(\eta \otimes \theta)_{\mathrm{a}, \varepsilon}=\sum_{\varepsilon_{1}+\varepsilon_{2}=\varepsilon} \eta_{K a, \varepsilon_{1}} \circ F \theta_{\mathrm{a}, \varepsilon_{2}}$ for each object $\mathrm{a} \in \mathcal{A}$ and $\varepsilon \in \mathbb{Z}_{2}$. The coherence maps are the obvious ones.

When working with monoidal supercategories, it will sometimes be convenient to use the following notation. Given objects $a$ and $b$ in a monoidal supercategory, we write $a b:=a \otimes b$. We will also write $a^{r}:=\underbrace{a \otimes \cdots \otimes a}_{r \text { times }}$.

\subsection{String Calculus}

There is a well-defined string calculus for strict monoidal supercategories discussed in $[\mathrm{BE} 1, \S 1.2]$. A morphism $f: \mathrm{a} \rightarrow \mathrm{b}$ is drawn as

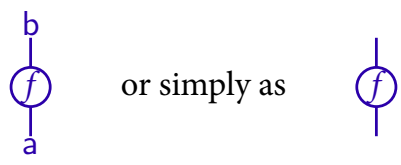

when the objects are left implicit. Notice that the convention used in this paper is to read diagrams from bottom to top. The products of morphisms $f \otimes g$ and $f \circ g$ are given by horizontal and vertical stacking respectively:

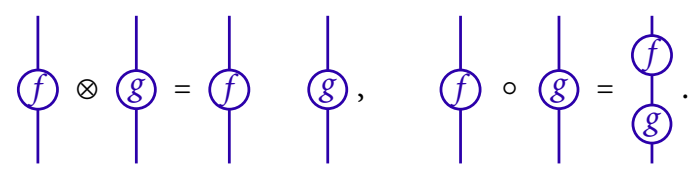

Pictures involving multiple products should be interpreted by first composing horizontally, then composing vertically. For example,

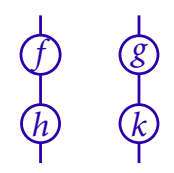

should be interpreted as $(f \otimes g) \circ(h \otimes k)$. In general, this is not the same as $(f \circ h) \otimes$ $(g \circ k)$, because of the super-interchange law:

$$
(f \otimes g) \circ(h \otimes k)=(-1)^{|g||h|}(f \circ h) \otimes(g \circ k) .
$$


In terms of string calculus, the super-interchange law implies that

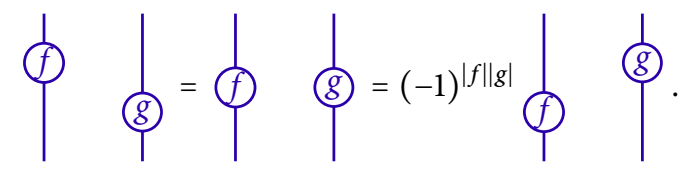

\subsection{Graded and Filtered Superspaces}

By a graded superspace we mean a $\mathbb{k}$-vector space $V$ equipped with a decomposition $V=\oplus_{(i, \varepsilon) \in \mathbb{Z} \times \mathbb{Z}_{2}} V_{i, \varepsilon}$. We write $V_{i}=V_{i, \overline{0}} \oplus V_{i, \overline{1}}$ for the elements of $V$ that are homogeneous of degree $i$. Given two graded superspaces $V$ and $W$, we write $\operatorname{Hom}_{\mathbb{k}}(V, W)_{i, \overline{0}}$ $\left(\right.$ resp. $\left.\operatorname{Hom}_{\mathbb{k}}(V, W)_{i, \overline{1}}\right)$ for the space of all $\mathbb{k}$-linear maps that map $V_{j, \varepsilon}$ to $W_{j+i, \varepsilon}$

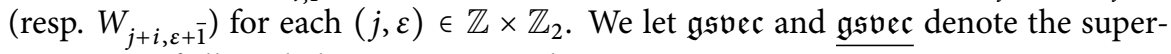
categories of all graded superspaces with

$$
\begin{aligned}
& \operatorname{Hom}_{\mathfrak{g s \mathfrak { s } \mathfrak { c }}}(V, W)=\bigoplus_{(i, \varepsilon) \in \mathbb{Z} \times \mathbb{Z}_{2}} \operatorname{Hom}_{\mathbb{k}}(V, W)_{i, \varepsilon}, \\
& \operatorname{Hom}_{\underline{\underline{g} \mathfrak{s} \mathfrak{v e c}}}(V, W)=\operatorname{Hom}_{\mathbb{k}}(V, W)_{0, \overline{0}} .
\end{aligned}
$$

There is a natural way to give $\mathfrak{g} \mathfrak{g} \mathfrak{v e c}$ (resp. $\underline{\mathfrak{g} \mathfrak{s} \mathfrak{e c})}$ the structure of a monoidal supercategory (resp. monoidal category) with

$$
(V \otimes W)_{i, \varepsilon}=\bigoplus_{(j, \eta) \in \mathbb{Z} \times \mathbb{Z}_{2}} V_{j, \eta} \otimes W_{i-j, \varepsilon+\eta} .
$$

By a filtered superspace we mean a superspace $V=V_{\overline{0}} \oplus V_{\overline{1}}$ equipped with a filtration $\cdots \subseteq V_{\leq i, \varepsilon} \subseteq V_{\leq i+1, \varepsilon} \subseteq \cdots$ such that $\bigcap_{(i, \varepsilon) \in \mathbb{Z} \times \mathbb{Z}_{2}} V_{\leq i, \varepsilon}=0$ and $\bigcup_{(i, \varepsilon) \in \mathbb{Z} \times \mathbb{Z}_{2}} V_{\leq i, \varepsilon}=V_{\varepsilon}$ for each $\varepsilon \in \mathbb{Z}_{2}$. We write $V_{\leq i}=V_{\leq i, \overline{0}} \oplus V_{\leq i, \overline{1}}$ for the elements of $V$ that are filtered degree $i$. Given two filtered superspaces $V$ and $W$, we write $\operatorname{Hom}_{\mathbb{k}}(V, W)_{\leq i, \overline{0}}$ (resp. $\left.\operatorname{Hom}_{\mathbb{k}}(V, W)_{\leq i, \overline{1}}\right)$ for the space of all $\mathbb{k}$-linear maps that map $V_{\leq j, \varepsilon}$ to $W_{\leq j+i, \varepsilon}$ (resp. $\left.W_{\leq j+i, \varepsilon+\overline{1}}\right)$ for each $(j, \varepsilon) \in \mathbb{Z} \times \mathbb{Z}_{2}$. We let $\mathfrak{f} \mathfrak{s v e c}$ and $\mathfrak{f} \mathfrak{s v e c}$ denote the supercategories of all filtered superspaces with

$$
\begin{aligned}
& \operatorname{Hom}_{\mathfrak{f} \mathfrak{s w e c}}(V, W)=\bigoplus_{(i, \varepsilon) \in \mathbb{Z} \times \mathbb{Z}_{2}} \operatorname{Hom}_{\mathbb{k}}(V, W)_{\leq i, \varepsilon}, \\
& \operatorname{Hom}_{\underline{\mathfrak{f} \mathfrak{s} \mathfrak{e c}}}(V, W)=\operatorname{Hom}_{\mathbb{k}}(V, W)_{\leq 0, \overline{0}} .
\end{aligned}
$$

There is a natural way to give $\mathfrak{f} \mathfrak{s v e c}(\operatorname{resp} . \underline{\mathfrak{f} \mathfrak{s e c})}$ the structure of a monoidal supercategory (resp. monoidal category) with

$$
(V \otimes W)_{\leq i, \varepsilon}=\bigoplus_{(j, \eta) \in \mathbb{Z} \times \mathbb{Z}_{2}} V_{\leq j, \eta} \otimes W_{\leq i-j, \varepsilon+\eta} .
$$

Every graded superspace $V$ can be viewed as a filtered superspace by setting $V_{\leq i, \varepsilon}=$ $\oplus_{j \leq i} V_{j, \varepsilon}$ for all $(i, \varepsilon) \in \mathbb{Z} \times \mathbb{Z}_{2}$. On the other hand, given a filtered superspace $V$, we write gr $V$ for the associated graded superspace with $(\operatorname{gr} V)_{i, \varepsilon}:=V_{\leq i, \varepsilon} / V_{\leq i-1, \varepsilon}$ for each $(i, \varepsilon) \in \mathbb{Z} \times \mathbb{Z}_{2}$. Given filtered superspaces $V$ and $W$, a map $f \in \operatorname{Hom}_{\mathbb{k}}(V, W)_{\leq i, \varepsilon}$ induces a map $\operatorname{gr}_{i, \varepsilon} f \in \operatorname{Hom}_{\mathbb{k}}(\operatorname{gr} V \text {, gr } W)_{i, \varepsilon}$ in an obvious way. 


\subsection{Graded and Filtered Supercategories}

By a graded (resp. filtered) supercategory we mean a category enriched in $\mathfrak{g} \mathfrak{s \mathfrak { v e c }}$ (resp. $\mathfrak{f} \mathfrak{s v e c}$ ). Similarly, a graded (resp. filtered) superfunctor is a functor enriched in $\mathfrak{g} \mathfrak{s v e c c}($ resp. $\mathfrak{f} \mathfrak{s} \mathfrak{e} \mathfrak{c})$. By a graded monoidal supercategory we mean a monoidal supercategory that is graded in such a way that $f \otimes g$ is homogeneous of degree $i+j$ whenever $f$ and $g$ are homogeneous of degree $i$ and $j$, respectively. Similarly, a filtered monoidal supercategory is a monoidal supercategory that is filtered in such a way that $f \otimes g$ has filtered degree $i+j$ whenever $f$ and $g$ have filtered degree $i$ and $j$ respectively.

Given a filtered supercategory $\mathcal{A}$, the associated graded supercategory gr $\mathcal{A}$ is the supercategory with the same objects as $\mathcal{A}$ and with $\operatorname{Hom}_{\mathrm{gr}} \mathcal{A}(\mathrm{a}, \mathrm{b}):=\operatorname{grHom}_{\mathcal{A}}(\mathrm{a}, \mathrm{b})$. The composition in gr $\mathcal{A}$ is induced from the composition in $\mathcal{A}$. Similarly, given a filtered superfunctor $F: \mathcal{A} \rightarrow \mathcal{B}$, we write gr $F: \operatorname{gr} \mathcal{A} \rightarrow \operatorname{gr} \mathcal{B}$ for the associated graded superfunctor defined in the obvious way.

For example, $\mathfrak{g} \mathfrak{s} \mathfrak{v} \mathfrak{c}$ and $\mathfrak{f} \mathfrak{s} \mathfrak{e} \mathfrak{c}$ are graded and filtered monoidal supercategories respectively. Note that gr $\mathfrak{f} \mathfrak{s v e c}$ and $\mathfrak{g s v e c}$ are not the same, but there is a faithful superfunctor $\Gamma: \operatorname{gr} \mathfrak{f} \mathfrak{s v e c} \rightarrow \mathfrak{g s v e c}$ that maps a filtered superspace to its associated graded superspace and maps $f+\operatorname{Hom}_{\mathbb{k}}(V, W)_{\leq i-1, \varepsilon} \in \operatorname{Hom}_{\mathbb{k}}(V, W)_{\leq i, \varepsilon} / \operatorname{Hom}_{\mathbb{k}}(V, W)_{\leq i-1, \varepsilon}$ to $\operatorname{gr}_{i, \varepsilon} f$.

\section{The Degenerate Affine Oriented Brauer-Clifford Supercategory}

In this section we define the monoidal supercategories $\mathcal{O B C}$ and $\mathcal{A O B C}$. First, however, we recall the definition of the supercategory $\mathcal{O B}$ from [BCNR]. Our definitions will make use of the string calculus for strict monoidal supercategories (see Section 2.3). In particular, each of the supercategories mentioned above admit a diagrammatic description. The objects in each of these supercategories are $\otimes$-generated by two objects denoted $\uparrow$ and $\downarrow$. Hence, the set of all objects can be identified with the set $\langle\uparrow, \downarrow\rangle$ of all finite words in the letters $\uparrow$ and $\downarrow$. The string diagrams for these supercategories will be made with oriented strings with an upward (resp. downward) string corresponding to the object $\uparrow$ (resp. $\downarrow$ ). For example, a diagram of the form

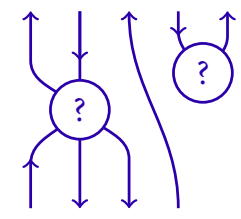

corresponds to a morphism $\uparrow \downarrow \downarrow \uparrow \rightarrow \uparrow \downarrow \uparrow \downarrow \uparrow$. We will describe classes of diagrams that give bases for the Hom-spaces of $\mathcal{O B C}$ and $\mathcal{A O B C}$. In this section we will show these diagrams indeed span the appropriate Hom-spaces. Proof that the diagrams are linearly independent will be given in Section 6 .

\subsection{The Oriented Brauer Category}

In [BCNR] the oriented Brauer category is defined diagrammatically and then a presentation is given in terms of generators and relations [BCNR, Theorem 1.1]. We take 
the latter description as our definition and view it as a supercategory concentrated in parity $\overline{0}$.

Definition 3.1 The oriented Brauer supercategory $\mathcal{O} \mathcal{B}$ is the $\mathbb{k}$-linear strict monoidal supercategory generated by two objects $\uparrow, \downarrow$ and three even morphisms $\bigcup: \mathbb{1} \rightarrow \uparrow \downarrow$, $\curvearrowleft: \downarrow \uparrow \rightarrow \mathbb{1}, \widehat{X}: \uparrow \uparrow \rightarrow \uparrow \uparrow$ subject to the following relations:

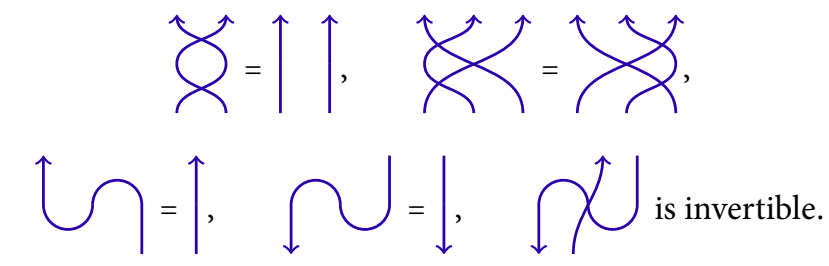

Note that the last relation is the assertion that there is another distinguished generator

$$
\varkappa: \uparrow \downarrow \rightarrow \downarrow \uparrow, \text { which is a two-sided inverse to } \Varangle:=凤 \text {. }
$$

We define rightward cups/caps and downward crossings in $\mathcal{O B}$ as follows:

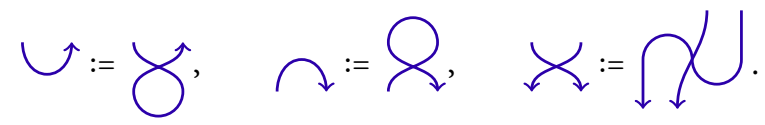

An oriented Brauer diagram with bubbles of type $\mathrm{a} \rightarrow \mathrm{b}$ is any string diagram obtained by stacking (vertically and horizontally) the defining generators of $O B B$ along with the diagrams (3.3) and (3.4) in such a way that the result can be interpreted as a morphism in $\operatorname{Hom}_{\mathcal{O B}}(a, b)$. For example, here are two oriented Brauer diagrams with bubbles of type $\downarrow \uparrow^{4} \downarrow^{2} \rightarrow \uparrow^{3} \downarrow^{2}$ :
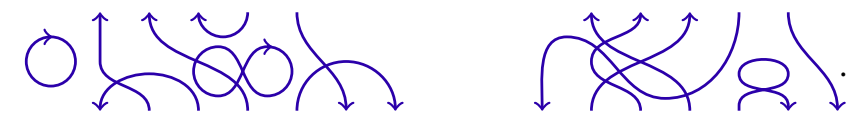

The term bubble refers to any component of such a diagram without an endpoint. In the examples above, the left diagram has two bubbles, whereas the right has none. An oriented Brauer diagram refers to an oriented Brauer diagram with bubbles that has no bubbles. We say that two oriented Brauer diagrams are equivalent if they are of the same type and one diagram can be obtained from the other by continuously deforming its strands, possibly moving them through other strands and crossings, but keeping endpoints fixed. Moreover, we say two oriented Brauer diagrams with bubbles are equivalent if they have the same number of bubbles and their underlying oriented Brauer diagrams (without bubbles) are equivalent. For example, the left (resp. right) diagram in (3.5) is equivalent to the following diagram on the left (resp. right):
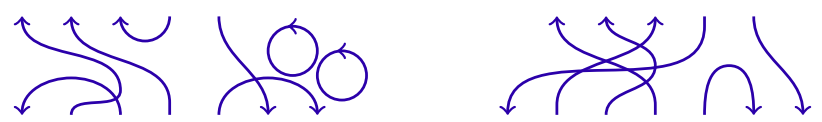

Of course, any morphism in $\mathcal{O B}$ can be realized as a $\mathbb{k}$-linear combination of oriented Brauer diagrams with bubbles. It follows from [BCNR, Theorem 1.1] that two 
oriented Brauer diagrams with bubbles represent the same morphism in $\mathcal{O B}$ if and only if they are equivalent. Moreover, the set of all equivalence classes of oriented Brauer diagrams with bubbles of type $a \rightarrow b$ is a basis for $\operatorname{Hom}_{\mathcal{O B}}(a, b)$.

As explained in [BCNR, $\mathbb{S} 1], \mathcal{O B}$ is a rigid symmetric monoidal supercategory. Briefly, the symmetric braiding on $\mathcal{O B}$ is the obvious one given on generating objects by the crossings $\checkmark, \prec, \prec$ Moreover, the generating objects are dual to one another with evaluation and coevaluation maps given by $\curvearrowleft, \curvearrowright$ and $\mathcal{U}, \smile$, respectively.

\subsection{The Oriented Brauer-Clifford Supercategory}

Adjoining "Clifford generators" to $\mathcal{O B}$ results in the following definition.

Definition 3.2 The oriented Brauer-Clifford supercategory $\mathcal{O B C}$ is the $\mathbb{k}$-linear strict monoidal supercategory generated by two objects $\uparrow, \downarrow$; three even morphisms $\mathcal{U}: \mathbb{1} \rightarrow \uparrow \downarrow, \curvearrowleft: \downarrow \uparrow \rightarrow \mathbb{1}, \widehat{X}: \uparrow \uparrow \rightarrow \uparrow \uparrow$; and one odd morphism $\hat{\$}: \uparrow \rightarrow \uparrow$ subject to (3.1), (3.2), and the following relations:

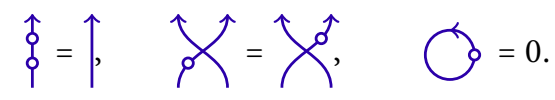

Note that the last relation in (3.6) makes use of the rightward cup defined by (3.4). We define the downward analogue of $\hat{p}$ as follows:

$$
\downarrow:=\{
$$

There is an obvious monoidal superfunctor $\mathcal{O B} \rightarrow \mathcal{O B} \mathcal{C}$ that allows us to view equivalence classes of oriented Brauer diagrams with bubbles as morphisms in $\mathcal{O B C}$. We define an oriented Brauer-Clifford diagram (resp. with bubbles) to be an oriented Brauer diagram (resp. with bubbles) with finitely many o's on its segments, where segment refers to a connected component of the diagram obtained when all crossings and local extrema are deleted. For example, here are two oriented Brauer-Clifford diagrams of type $\downarrow \uparrow \uparrow \uparrow \downarrow \downarrow \rightarrow \uparrow \downarrow \uparrow \downarrow \uparrow \downarrow:$
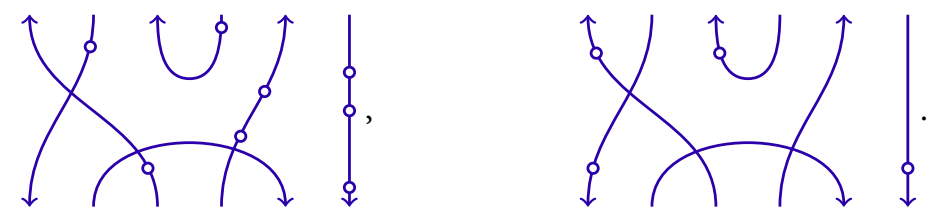

With (3.7) in mind, we can interpret any oriented Brauer-Clifford diagram with bubbles as a morphism in $\mathcal{O B C}$.

Since $\hat{\phi}$ is odd, we must keep the super-interchange law in mind when sliding o's along strands. For example, by (2.1) we have

$$
\uparrow \mathfrak{l}=\uparrow \downarrow=-\uparrow \downarrow .
$$


For a more complicated example, in (3.8) numerous signs occur if one tries to rewrite the left-hand diagram into the right-hand diagram. For example, the $\circ$ in the upper left corner of the right-hand diagram could be obtained by sliding the corresponding ○ in the left-hand digram upward along its strand. In doing so it would have to pass six of the other o's in the left-hand diagram. Hence, this move would incur a cost of scaling by $(-1)^{6}$. However, the following result shows that o's are allowed to freely slide along cups/caps and pass through crossings.

Proposition 3.3 The following relations hold in $\mathrm{OBC}$ :

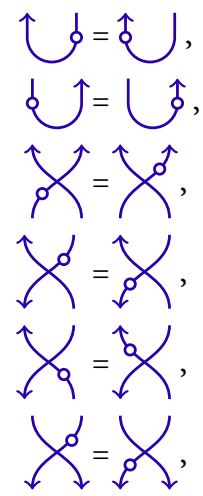

$$
\begin{aligned}
& \bigcap=\emptyset \text {, } \\
& \bigcap=\bigcap \text {, } \\
& \chi_{q}=\hat{\gamma} \text {. } \\
& \xi=\chi_{q}, \\
& Y=X^{0} \text {, } \\
& \downarrow=\chi_{q} \text {. }
\end{aligned}
$$

Proof The left side of (3.9) is verified below; the right side is similar.

$$
\uparrow \stackrel{(3.7)}{=}\lceil\hat{U}=\uparrow \Omega \stackrel{(3.2)}{=}\} .
$$

Next, we prove (3.11)-(3.14). In (3.11)- (3.14) the right equality is obtained from the left by composing on top and bottom with $\chi, \succ$, and $\Varangle$, respectively. The left side of (3.11) is one of the defining relations of $O \mathcal{B C}$. The left side of (3.12) is verified below:

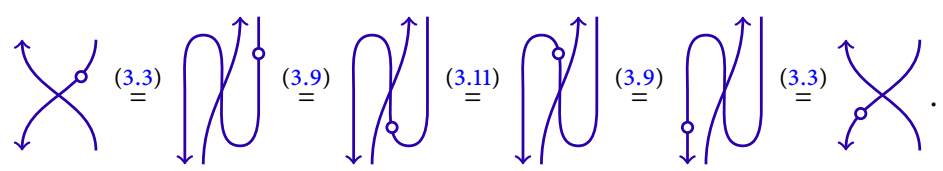

Similarly, the left side of (3.13) (resp. (3.14)) follows from (3.3) (resp. (3.4)) and the left side of (3.11) (resp. (3.12)).

Finally, we verify the left side of (3.10) below.

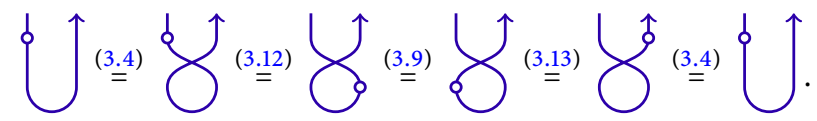

The right side of (3.10) is similar.

Next, we will flesh out more of the diagrammatic nature of $\mathcal{O B C}$. However, before doing so we pause to point out that (3.11)-(3.14) imply the symmetric braiding on $\mathcal{O B}$ extends to a supernatural isomorphism for $\mathcal{O B C}$. In particular, $\mathcal{O B C}$ inherits the structure of a rigid symmetric monoidal supercategory from $\mathcal{O B}$. 
We have a downward analogue of the left relation in (3.6):

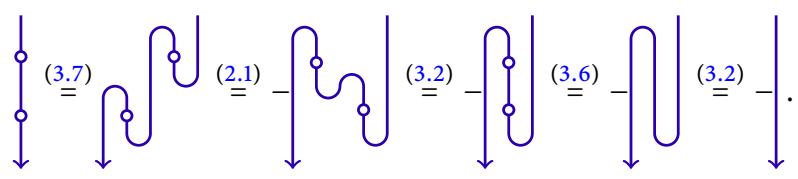

In particular, it follows that every oriented Brauer-Clifford diagram with bubbles can be reduced, up to sign, to a diagram with the same underlying oriented Brauer diagram and at most one on each strand. For example, the two diagrams in (3.8) represent the same morphism in $\mathcal{O B C}$.

Additionally, we have an even analogue of the right relation in (3.6). Indeed,

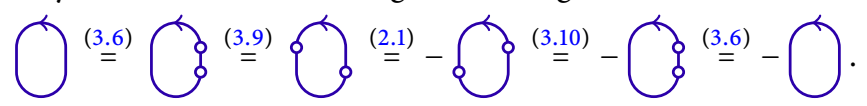

Since char $\mathbb{k} \neq 2$, the computation above implies that

$$
\circlearrowleft=0 \text {. }
$$

It follows that any oriented Brauer-Clifford diagram with at least one bubble reduces to zero in $\mathcal{O B C}$. Hence, the Hom-spaces in $\mathcal{O B C}$ are spanned by oriented BrauerClifford diagrams (without bubbles) that have at most one o on each strand. Next, we refine this spanning set.

We say that an oriented Brauer-Clifford diagram is normally ordered if

$\diamond$ it has at most one $\circ$ on each strand; all o's are on outward-pointing boundary segments (i.e., segments that intersect the boundary at a point that is directed out of the picture);

$\diamond$ all o's that occur on upwardly oriented segments are positioned at the same height; similarly, all o's that occur on downwardly oriented segments are positioned at the same height.

For example, in (3.8) the diagram on the right is normally ordered, and the one on the left is not. We say that two normally ordered oriented Brauer-Clifford diagrams are equivalent if their underlying oriented Brauer diagrams are equivalent (see Section 3.1) and their corresponding strands have the same number of o's on them. Note that two equivalent normally ordered oriented Brauer-Clifford diagrams correspond to the same morphism in $\mathcal{O B C}$. Moreover, it follows from the discussion above that the Hom-spaces in $\mathcal{O B C}$ are spanned by normally ordered oriented Brauer-Clifford diagrams. In fact, we have the following theorem.

Theorem 3.4 For any $\mathrm{a}, \mathrm{b} \in\langle\uparrow, \downarrow\rangle$, the superspace $\operatorname{Hom}_{\mathcal{O B}}(\mathrm{a}, \mathrm{b})$ has basis given by equivalence classes of normally ordered oriented Brauer-Clifford diagrams of type $\mathrm{a} \rightarrow \mathrm{b}$.

It is possible to give a straightforward combinatorial proof of Theorem 3.4. However, we omit such a proof, since Theorem 3.4 is a consequence of our basis theorem for $\mathcal{A O B C}$ as explained in Section 3.4.

Meanwhile, let us point the following consequence of Theorem 3.4. For $r \geq 1$, let $C_{r}$ denote the Clifford algebra generated by $c_{1}, \ldots, c_{r}$, subject to the relations $c_{i}^{2}=1$ and $c_{i} c_{j}=-c_{j} c_{i}$ for $i \neq j$. We view $C_{r}$ as a superalgebra by declaring the generators to 
be odd. Let $\mathbb{k} \Sigma_{r}$ denote the group algebra of the symmetric group on $r$ letters viewed as a superalgebra concentrated in parity $\overline{0}$. Then the (finite) Sergeev superalgebra is

$$
\operatorname{Ser}_{r}=C_{r} \otimes \mathbb{k} \Sigma_{r}
$$

as a superspace with $C_{r} \otimes 1 \cong C_{r}$ and $1 \otimes \mathbb{k} \Sigma_{r} \cong \mathbb{k} \Sigma_{r}$ as subsuperalgebras and with mixed relation $w c_{i}=c_{w(i)} w$ for $i=1, \ldots, r$ and all $w \in \Sigma_{r}$.

Corollary 3.5 For $r \geq 1$, $\operatorname{End}_{\mathcal{O B C}}\left(\uparrow^{r}\right) \cong \operatorname{Ser}_{r}$.

Proof The Sergeev superalgebra is generated by $s_{1}, \ldots, s_{r-1}, c_{1}, \ldots, c_{r}$ subject to $s_{i}^{2}=1, s_{i} s_{j}=s_{j} s_{i}$ if $|i-j|>1, s_{i} s_{i+1} s_{i}=s_{i+1} s_{i} s_{i+1}, c_{i}^{2}=1, c_{i} c_{j}=-c_{j} c_{i}$ if $i \neq j$, and $s_{i} c_{i}=c_{i+1} s_{i}$ for all admissible $1 \leq i, j \leq r$. From this it follows that there is a well-defined superalgebra map

$$
\varphi: \operatorname{Ser}_{r} \longrightarrow \operatorname{End}_{\mathcal{O B C}}\left(\uparrow^{r}\right)
$$

given by

$$
\varphi\left(s_{i}\right)=\uparrow^{r-i-1} \chi \uparrow^{i-1} \text { and } \varphi\left(c_{i}\right)=\uparrow^{r-1} \hat{\Phi}^{i-1} .
$$

Using Theorem 3.4, it is straightforward to see that $\varphi$ is an isomorphism. In particular, a direct count shows that there are $2^{r}(r !)$ normally ordered oriented Brauer diagrams of type $\uparrow^{r} \rightarrow \uparrow^{r}$, which matches the dimension of $\operatorname{Ser}_{r}$.

More generally, given nonnegative integers $r, s$, let $B C_{r, s}$ denote the walled BrauerClifford superalgebra. This superalgebra was introduced in [JK] where it is denoted $\vec{B}_{r, s}$ and called the walled Brauer superalgebra. Our notation and terminology is chosen so as to be consistent with that of $[\mathrm{BGH}+]$. In the next result, we assume that $\mathbb{k}$ contains $\sqrt{-1}$.

Corollary 3.6 Let a be a word consisting of $r \uparrow$ 's and $s \downarrow$ 's. Then

$$
\operatorname{End}_{\mathcal{O B C}}(\mathbf{a}) \cong B C_{r, s}
$$

as superalgebras.

Proof We first note that if $\mathbf{a}$ and $\mathbf{b}$ are two words with $r \uparrow$ 's and $s \downarrow$ 's, then

$$
\operatorname{End}_{\mathcal{O B C}}(\mathbf{a}) \cong \operatorname{End}_{\mathcal{O B}}(\mathbf{b}) .
$$

The isomorphism is given by applying suitable symmetric braidings for $\mathcal{O B C}$. Consequently, assume $\mathbf{a}=\downarrow^{s} \uparrow^{r}$. By [JK, Theorem 5.1], $B C_{r, s}$ is generated by even generators $s_{1}, \ldots, s_{r-1}, s_{r+1}, \ldots, s_{r+s-1}, e_{r, r+1}$ and odd generators $c_{1}, \ldots, c_{r+s}$ subject to an explicit set of relations. By checking relations, we see that there is a superalgebra map 
$\alpha: B C_{r, s} \rightarrow \operatorname{End}_{\mathcal{O B C}}\left(\downarrow^{s} \uparrow^{r}\right)$ given by

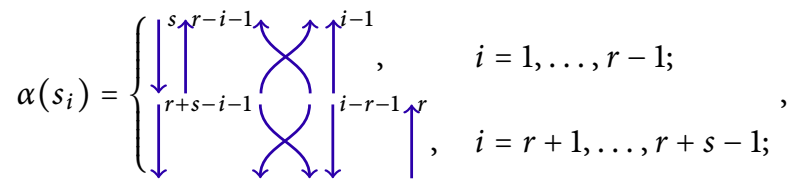

$$
\begin{aligned}
& \alpha\left(e_{r, r+1}\right)=\left.\downarrow^{s-1} \smile\right|^{r-1}, \\
& \alpha\left(c_{i}\right)= \begin{cases}\left.\left.\sqrt{-1}\right|^{s}\right|^{r-i} \uparrow \uparrow^{i-1}, & i=1, \ldots, r ; \\
\left.\sqrt{-1}\rfloor^{r+s-i} \downarrow\right\rfloor^{i-r-1} \uparrow^{r}, & i=r+1, \ldots, r+s .\end{cases}
\end{aligned}
$$

As in the previous theorem, one can use Theorem 3.4 to verify that $\alpha$ is an isomorphism. We leave the details to the reader.

\subsection{The Supercategory $\mathcal{A O B} \mathcal{B}$}

We now introduce an affine version of $\mathcal{O B C}$.

Definition 3.7 The degenerate affine oriented Brauer-Clifford supercategory $\mathcal{A O B} \mathcal{B}$ is the $\mathbb{k}$-linear strict monoidal supercategory generated by two objects $\uparrow, \downarrow$; four even morphisms $\bigcup: \mathbb{1} \rightarrow \uparrow \downarrow \curvearrowleft: \downarrow \uparrow \rightarrow \mathbb{1}, \widehat{X}: \uparrow \uparrow \rightarrow \uparrow \uparrow, \hat{\mathbf{\phi}}: \uparrow \rightarrow \uparrow$; and one odd morphism $\hat{\$}: \uparrow \rightarrow \uparrow$ subject to (3.1), (3.2), (3.6), and the following relations:

$$
\$=-\$, \zeta-\nmid=\uparrow \uparrow-\uparrow \uparrow .
$$

We define the downward analogue of 1 as follows:

$$
\downarrow:=\downarrow \text {. }
$$

We define a dotted oriented Brauer-Clifford diagram (resp. with bubbles) to be an oriented Brauer diagram (resp. with bubbles) with finitely many o's and 's on its segments. For example, (1.1) shows two dotted oriented Brauer-Clifford diagrams with bubbles of type $\downarrow^{2} \uparrow^{2} \rightarrow \downarrow^{2} \uparrow^{3} \downarrow$. Given a nonnegative integer $k$, we will draw a $\bullet$ labeled by $k$ to denote $k$ 's on a strand, that is, the vertical composition of $k \$$ 's or $k$ 's. For example, the diagram on the right side of (1.1) could have been drawn as

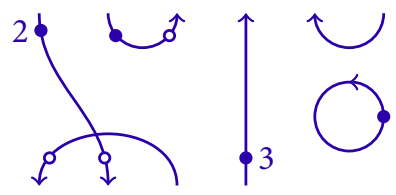

We can interpret any dotted oriented Brauer-Clifford diagram with bubbles as a morphism in $\mathcal{A O B C}$, and the Hom-spaces in $\mathcal{A O B} \mathcal{B}$ are certainly spanned by all dotted oriented Brauer-Clifford diagrams with bubbles. For all $\mathrm{a}, \mathrm{b} \in\langle\uparrow, \downarrow\rangle$ and each $k \in \mathbb{Z}$, 
we let $\operatorname{Hom}_{\mathcal{A O B C}}(\mathrm{a}, \mathrm{b})_{\leq k, \overline{0}}\left(\operatorname{resp} . \operatorname{Hom}_{\mathcal{A O B C}}(\mathrm{a}, \mathrm{b})_{\leq k, \overline{1}}\right)$ denote the $\mathbb{k}$-span of all dotted oriented Brauer-Clifford diagrams with bubbles of type $\mathrm{a} \rightarrow \mathrm{b}$ having at most $k$ $\therefore$ 's and an even (resp. odd) number of o's. This gives $\mathcal{A O B C}$ the structure of a filtered monoidal supercategory (see Section 2.5). Given a dotted oriented Brauer-Clifford diagram with bubbles, $d$, we will write $\operatorname{deg}(d)$ for the number of $\bullet$ 's appearing in $d$. For example, $\operatorname{deg}(d)=7$ when $d$ is either of the diagrams in (1.1).

Definition 3.8 A dotted oriented Brauer-Clifford diagram with bubbles is normally ordered if the following hold:

$\diamond$ removing all bubbles and all $\bullet$ 's results in a normally ordered oriented BrauerClifford diagram;

$\diamond$ each bubble has zero o's and an odd number of $\bullet$ 's, are crossing-free, counterclockwise, and there are no other strands shielding it from the rightmost edge of the picture;

$\diamond$ each $\bullet$ is either on a bubble or on an inward-pointing boundary segment;

$\diamond$ whenever $\mathrm{a} \bullet$ and a $\circ$ appear on a segment that is both inward and outwardpointing, the $\circ$ appears ahead of the $\bullet$ in the direction of the orientation.

For example, in (1.1) the diagram on the right is normally ordered and the one on the left is not. We say that two normally ordered dotted oriented Brauer-Clifford diagrams with bubbles are equivalent if their underlying oriented Brauer diagrams with bubbles are equivalent and their corresponding strands have the same number of o's and $\bullet$ 's. We can now state our main result.

Theorem 3.9 For any $\mathrm{a}, \mathrm{b} \in\langle\uparrow, \downarrow\rangle$, the superspace $\operatorname{Hom}_{\mathcal{A O B C}}(\mathrm{a}, \mathrm{b})$ has basis given by equivalence classes of normally ordered dotted oriented Brauer-Clifford diagrams with bubbles of type $\mathrm{a} \rightarrow \mathrm{b}$.

\subsection{Consequences of Theorem 3.9 for $\mathcal{O B C}$}

For this subsection we assume Theorem 3.9. Let $\mathcal{O B}(0)$ denote the quotient of $\mathcal{O B}$ obtained by requiring (3.16). Then the Hom-spaces in $\mathcal{O B}(0)$ have a basis given by equivalence classes of oriented Brauer diagrams without bubbles. Moreover, there are obvious monoidal superfunctors

$$
\mathcal{O B}(0) \rightarrow \mathcal{O B C} \rightarrow \mathcal{A O B C}
$$

We saw in Section 3.2 that the Hom-spaces in $\mathcal{O B C}$ are spanned by normally ordered oriented Brauer-Clifford diagrams. It follows from Theorem 3.9 that the image of the equivalence classes of normally ordered oriented Brauer-Clifford diagrams under (3.18) are linearly independent in $\mathcal{A O B} \mathcal{C}$, whence they are linearly independent in OBC. Theorem 3.4 follows. Moreover, it follows that the monoidal superfunctors in (3.18) are both faithful.

\subsection{Normally Ordered Diagrams Span}

In Section 3.2 we showed that normally ordered oriented Brauer-Clifford diagrams span the Hom-spaces in $O \mathcal{O B}$. The goal of this subsection is to show that normally 
ordered dotted oriented Brauer-Clifford diagrams with bubbles span the Hom-spaces in $\mathcal{A O B C}$. We start with an analogue of Proposition 3.3 for $\mathcal{A O B C}$. In the proof, we will make use of Proposition 3.3 without reference.

Proposition 3.10 The following relations hold in $\mathcal{A O B C}$ :

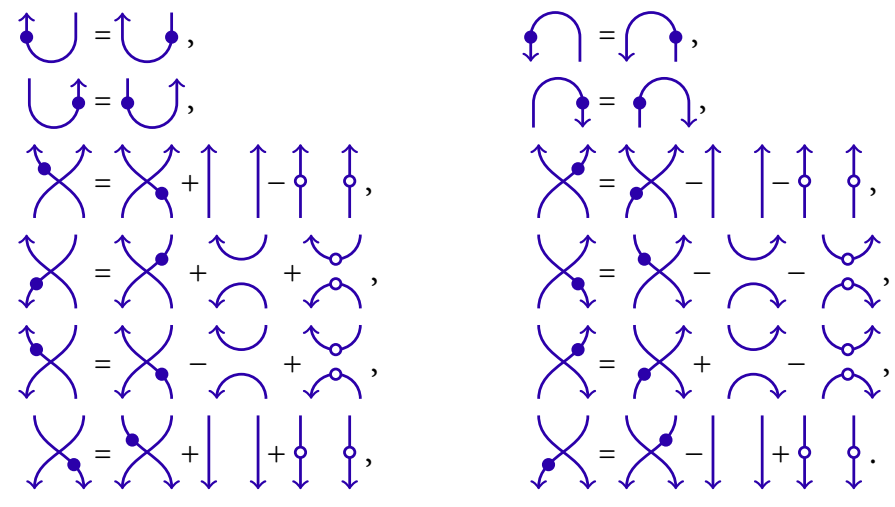

Proof The proof of (3.19) is similar to that of (3.9). In (3.21), (3.22), (3.23), and (3.24) the right equality is obtained from the left by composing on top and bottom with ,, , and $\nsucc$ respectively. The left side of (3.21) is one of the defining relations of $\mathcal{A O B C}$. The left side of (3.22) is verified below:

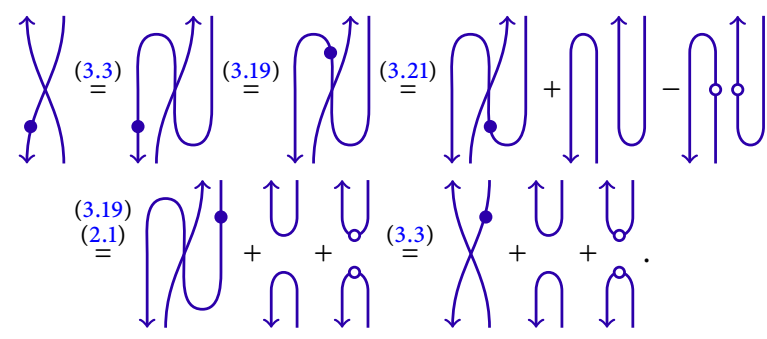

Proofs for the left equalities in (3.23) and (3.24) are similar. Finally, we verify the right side of (3.20) below:

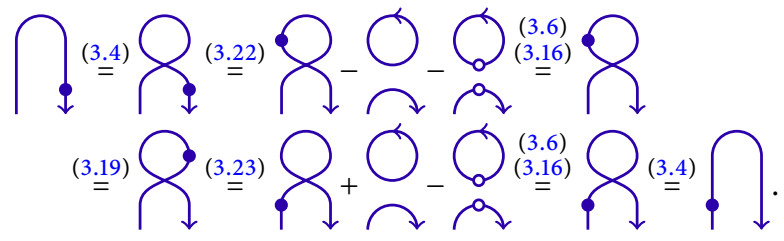

The left side of (3.20) is similar.

Note that (3.9)-(3.10) and (3.19)-(3.20) allow us to draw o's and 's on local extrema of strands in dotted oriented Brauer-Clifford diagrams with bubbles without ambiguity. We will do so whenever convenient. 
We have a downward analogue of the left relation in (3.17):

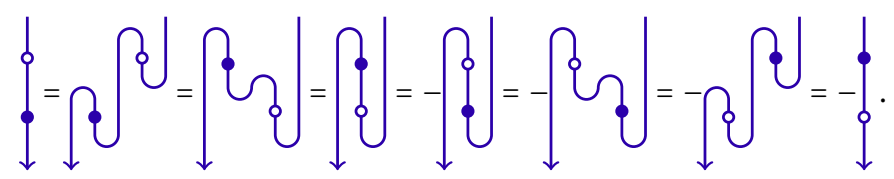

Before proving that normally ordered dotted oriented Brauer-Clifford diagrams with bubbles span arbitrary Hom-spaces in $\mathcal{A O B} \mathcal{B}$, we first consider diagrams of type $\mathbb{1} \rightarrow \mathbb{1}$. In this case we must show the superalgebra $\operatorname{End}_{\mathcal{A O B \mathcal { B }}}(\mathbb{1})$ is generated by $\Delta_{1}, \Delta_{3}, \Delta_{5}, \ldots$, where

$$
\Delta_{k}=\bigcirc_{k} .
$$

First, note the defining relations of $\mathcal{A O B C}$ (and the similar relations on non-upward pointed strands implied by these) along with Propositions 3.3 and 3.10 can be used to express any dotted oriented Brauer-Clifford diagram with bubbles of type $\mathbb{1} \rightarrow \mathbb{1}$ in terms of crossing-free un-nested bubbles. These relations allow one to pull apart nested bubbles, pass o's through crossings, cups, and caps as needed, and 's through crossings at the cost of additional diagrams with fewer $\bullet$ 's. Repeated moves of this sort can be used to rewrite any diagram of type $\mathbb{1} \rightarrow \mathbb{1}$ into the claimed form. For example,

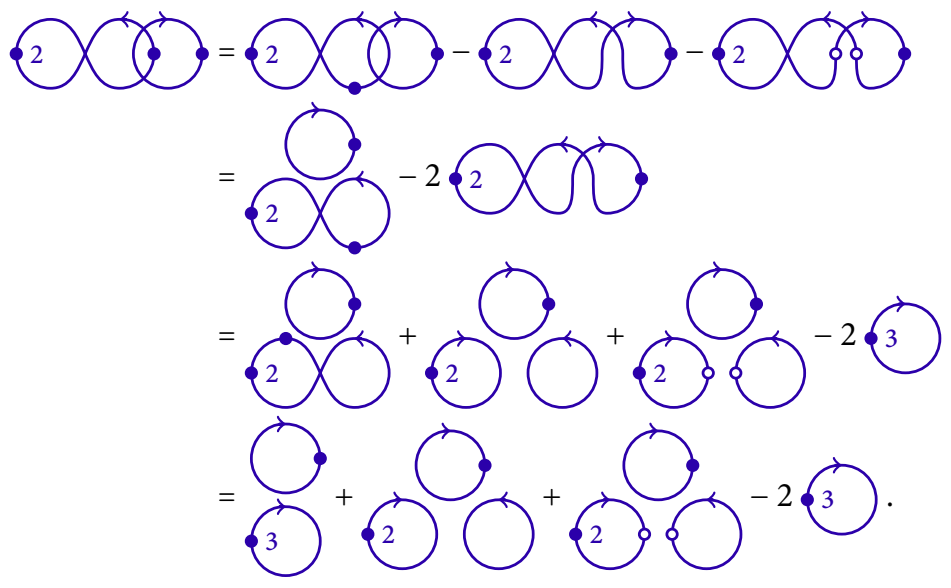

Moreover, we can express any clockwise bubble in terms of counterclockwise ones using computations similar to the following:

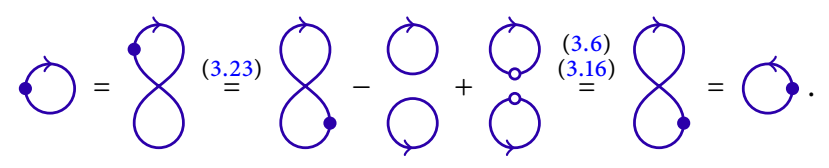

Remark 3.11 (Compare with [BCNR, Remark 1.3]) Set $\Delta_{-1}:=\mathbb{1}, \Delta_{-1}^{\prime}:=-\mathbb{1}$, and

$$
\Delta_{k}^{\prime}:=k \bigcirc
$$


for all integers $k \geq 0$. The computation above shows $\Delta_{-1} \Delta_{1}^{\prime}+\Delta_{1} \Delta_{-1}^{\prime}=0$. A similar computation can be used to show

$$
\sum_{0 \leq i \leq \frac{k+1}{2}} \Delta_{2 i-1} \Delta_{k-2 i}^{\prime}=0
$$

whenever $k$ is a nonnegative odd integer.

Now, using (3.6), (3.15), (3.17), and (3.25) we can reduce the number of o's on any bubble to zero or one. Finally, the following proposition shows that the only nonzero counterclockwise bubbles with at most one $\circ$ are $\Delta_{1}, \Delta_{3}, \Delta_{5}, \ldots$, whence they generate $\operatorname{End}_{\mathcal{A O B} \mathcal{B}}(\mathbb{1})$.

Proposition 3.12 The following relations hold in $\mathcal{A O B C}$ for any nonnegative integer $k$ :

$$
\bigcup_{k=0}, \bigcirc_{2 k}=0
$$

Proof The left relation when $k=0$ follows from the right side of (3.6). If $k>0$, the left relation follows from the calculation below since char $\mathbb{k} \neq 2$ :

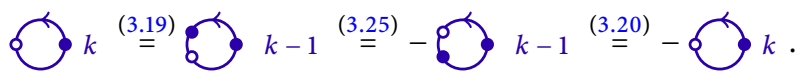

The right relation follows from the calculation below:

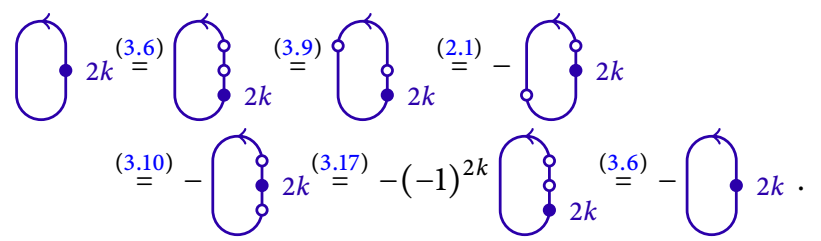

The following lemma implies that normally ordered oriented Brauer-Clifford diagrams with bubbles span the Hom-spaces of $\mathcal{A O B C}$.

Lemma 3.13 For any $\mathrm{a}, \mathrm{b} \in\langle\uparrow, \downarrow\rangle$, the superspace $\operatorname{Hom}_{\mathcal{A O B} \mathcal{B}}(\mathrm{a}, \mathrm{b})_{\leq k}$ is equal to the $\mathbb{k}$-span of all equivalence classes of normally ordered dotted oriented Brauer-Clifford diagrams with bubbles of type $\mathrm{a} \rightarrow \mathrm{b}$ with at most $k \bullet '$ '.

Proof Let $d$ denote a dotted oriented Brauer-Clifford diagram with bubbles with $\operatorname{deg}(d) \leq k$. Let $d^{\prime}$ denote the diagram obtained from $d$ as follows. First, remove pairs of o's appearing on the same strand until each strand has at most one o. Next, freely slide each bubble to the right side of the picture and redraw them so that they are crossing-free and counterclockwise, without changing the number of o's and 's appearing on each bubble. Finally, on every non-bubble strand, freely slide all o's (resp. 's) until they lie on an outward-pointing (resp. inward-pointing) segment. For example, if $d$ is the diagram on the left side of (1.1), then $d^{\prime}$ is the diagram on the right. It follows from Proposition 3.12 that $d^{\prime}$ is either zero or normally ordered with $\operatorname{deg}\left(d^{\prime}\right)=\operatorname{deg}(d)$. Moreover, by Propositions 3.3 and 3.10 along with (3.6), (3.15), and (3.25), we have $d= \pm d^{\prime}+d^{\prime \prime}$ where $d^{\prime \prime}$ is a linear combination of dotted oriented 
Brauer-Clifford diagrams with bubbles each of which having fewer than $\operatorname{deg}(d) \bullet$ 's. The result now follows by inducting on $\operatorname{deg}(d)$.

\section{Connection to Representations of Lie Superalgebras of Type Q}

We next explain how the supercategories $\mathcal{O B C}$ and $\mathcal{A O B C}$ are connected with the representations of the Lie superalgebras of type $Q$. In what follows, we assume that $\sqrt{-1}$ is an element of $\mathbb{k}$. This is only for convenience. In particular, the arguments in Section 6.2 imply the basis theorems hold in general.

\subsection{The Lie Superalgebra $\mathfrak{q}$}

Fix a $\mathbb{k}$-superspace $V=V_{\overline{0}} \oplus V_{\overline{1}}$ with $\operatorname{dim}_{\mathbb{k}}\left(V_{\overline{0}}\right)=\operatorname{dim}_{\mathbb{k}}\left(V_{\overline{1}}\right)=n$. Fix a homogeneous basis $v_{1}, \ldots, v_{n}, v_{\overline{1}}, \ldots, v_{\bar{n}}$ with $\left|v_{i}\right|=\overline{0}$ and $\left|v_{\bar{i}}\right|=\overline{1}$ for $i=1, \ldots, n$. We write $I$ for the index set $\{1, \ldots, n, \overline{\overline{1}}, \ldots, \bar{n}\}$ and $I_{0}$ for the index set $\{1, \ldots, n\}$. We adopt the convention that $\bar{i}=i$ for all $i \in I$. Let $c: V \rightarrow V$ be the odd linear map given by $c\left(v_{i}\right)=(-1)^{\left|v_{i}\right|} \sqrt{-1} v_{\bar{i}}$ for all $i \in I$.

The vector space of all linear endomorphisms of $V, \mathfrak{g l}(V)$, is naturally $\mathbb{Z}_{2}$-graded as in Section 2.1. Furthermore, $\mathfrak{g l}(V)$ is a Lie superalgebra under the graded commutator bracket; this, by definition, is given by $[x, y]=x y-(-1)^{|x||y|} y x$ for all homogeneous $x, y \in \mathfrak{g l}(V)$. For $i, j \in I$, we write $e_{i, j} \in \mathfrak{g l}(V)$ for the linear map $e_{i, j}\left(v_{k}\right)=\delta_{j, k} v_{i}$. These are the matrix units and they form a homogeneous basis for $\mathfrak{g l}(V)$ with $\left|e_{i, j}\right|=\left|v_{i}\right|+\left|v_{j}\right|$.

By definition, $\mathfrak{q}(V)$ is the Lie subsuperalgebra of $\mathfrak{g l}(V)$ given by

$$
\mathfrak{q}(V)=\{x \in \mathfrak{g l}(V) \mid[x, c]=0\} .
$$

Then $\mathfrak{q}(V)$ has a homogenous basis given by $e_{i, j}^{\overline{0}}:=e_{i, j}+e_{\bar{i}, \bar{j}}$ and $e_{i, j}^{\overline{1}}:=e_{\bar{i}, j}+e_{i, \bar{j}}$ for $1 \leq i, j \leq n$. Set

$$
\widetilde{e}_{i, j}^{\overline{0}}:=e_{i, j}-e_{\bar{i}, \bar{j}} \text { and } \quad \bar{e}_{i, j}^{\overline{1}}:=e_{\bar{i}, j}-e_{i, \bar{j}}
$$

for $1 \leq i, j \leq n$. These are homogeneous elements of $\mathfrak{g l}(V)$ and, together with our basis for $\mathfrak{q}(V)$, provide a homogeneous basis for $\mathfrak{g l}(V)$. Note that $\left|\widetilde{e}_{i, j}^{\mathfrak{z}}\right|=\left|e_{i, j}^{\varepsilon}\right|=\varepsilon$ for all $1 \leq i, j \leq n$ and $\varepsilon \in \mathbb{Z}_{2}$.

Let $U(\mathfrak{q})$ denote the universal enveloping superalgebra of the Lie superalgebra $\mathfrak{q}=$ $\mathfrak{q}(V)$. The superalgebra $U(\mathfrak{q})$ has a homogeneous PBW basis given by all ordered monomials in the elements $\left(e_{i, j}^{\varepsilon}\right)^{r}$ (with $1 \leq i, j \leq n$, and $r \geq 1$ if $\varepsilon=\overline{0}$ or $r=0,1$ if $\varepsilon=\overline{1})$. Set $U(\mathfrak{h}), U(\mathfrak{n})$, and $U\left(\mathfrak{n}^{-}\right)$, respectively, to be the subsuperalgebras generated by

$\left\{e_{i, i}^{\varepsilon} \mid i=1, \ldots, n, \varepsilon \in \mathbb{Z}_{2}\right\},\left\{e_{i, j}^{\varepsilon} \mid 1 \leq i<j \leq n, \varepsilon \in \mathbb{Z}_{2}\right\},\left\{e_{i, j}^{\varepsilon} \mid 1 \leq j<i \leq n, \varepsilon \in \mathbb{Z}_{2}\right\}$.

The PBW basis implies that there is a triangular decomposition $U(\mathfrak{q})=U\left(\mathfrak{n}^{-}\right) \otimes$ $U(\mathfrak{h}) \otimes U(\mathfrak{n})$.

A $U(\mathfrak{q})$-supermodule is a $\mathbb{k}$-superspace $M=M_{\overline{0}} \oplus M_{\overline{1}}$ with an action by $U(\mathfrak{q})$ that respects the $\mathbb{Z}_{2}$-grading in that $U(\mathfrak{q})_{\varepsilon} M_{\varepsilon^{\prime}} \subseteq M_{\varepsilon+\varepsilon^{\prime}}$ for all $\varepsilon, \varepsilon^{\prime} \in \mathbb{Z}_{2}$. In particular, the superspace $V$ defined above is naturally a $U(\mathfrak{q})$-supermodule. A supermodule homomorphism is a $\mathbb{k}$-linear map $f: M \rightarrow N$ that satisfies $f(a m)=(-1)^{|f||a|} a f(m)$ for 
all homogeneous $a \in U(\mathfrak{q})$ and $m \in M$. Note that homomorphisms are not assumed to preserve parity. However, Hom-spaces are naturally $\mathbb{Z}_{2}$-graded as in Section 2.1. Let $U(\mathfrak{q})$-smod denote the supercategory of all $U(\mathfrak{q})$-supermodules. We will write $\operatorname{Hom}_{U(\mathfrak{q})}\left(M, M^{\prime}\right)=\operatorname{Hom}_{U}(\mathfrak{q})-\operatorname{smod}\left(M, M^{\prime}\right)$.

The $\mathbb{k}$-superalgebra $U(\mathfrak{q})$ is a Hopf superalgebra. In particular, given $U(\mathfrak{q})$-supermodules $M$ and $M^{\prime}$, the action of a homogeneous $x \in \mathfrak{q} \subseteq U(\mathfrak{q})$ on $M \otimes M^{\prime}$ is given by $x .\left(m \otimes m^{\prime}\right)=(x . m) \otimes m^{\prime}+(-1)^{|x||m|} m \otimes\left(x . m^{\prime}\right)$ for homogeneous $m \in M$ and $m^{\prime} \in M^{\prime}$. The unit object is given by viewing $\mathbb{k}$ as a superspace concentrated in parity $\overline{0}$ and with trivial $U(\mathfrak{q})$ action. The symmetric braiding is given by the graded flip map. Thus, $U(\mathfrak{q})$-smod is a symmetric braided monoidal supercategory.

The antipode $\sigma: U(\mathfrak{q}) \rightarrow U(\mathfrak{q})$ is given by $\sigma(x)=-x$ for $x \in \mathfrak{q}$. Using the antipode, each finite-dimensional $U(\mathfrak{q})$-supermodule $M$ has a dual given by $M^{*}=$ $\operatorname{Hom}_{\mathbb{k}}(M, \mathbb{k})$ with the evaluation and coevaluation maps given by the same formulas as for superspaces (see Section 2.1). In particular, $V$ is finite-dimensional and so admits a dual, $V^{*}$.

\subsection{Mixed Schur-Weyl-Sergeev Duality}

As Theorem 3.4 and Corollary 3.5 will be used to prove the following results, let us point out that this section is not used in the proof of Theorems 3.9 and 3.4 and Corollary 3.5 .

There is a monoidal superfunctor

$$
\Phi: \mathcal{O B C} \longrightarrow U(\mathfrak{q}) \text {-smod }
$$

mapping the objects $\uparrow, \downarrow$ to the superspaces $V, V^{*}$, respectively, and defined on morphisms by

$$
\begin{aligned}
& \Phi(\smile): \mathbb{1} \longrightarrow V \otimes V^{*}, \quad \quad 1 \longmapsto \sum_{i \in I} v_{i} \otimes v_{i}^{*}, \\
& \Phi(\curvearrowleft): V^{*} \otimes V \longrightarrow \mathbb{1}, \quad f \otimes v \longmapsto f(v), \\
& \Phi(\widehat{\chi}): V \otimes V \longrightarrow V \otimes V, \quad u \otimes v \longmapsto(-1)^{|u||v|} v \otimes u,
\end{aligned}
$$

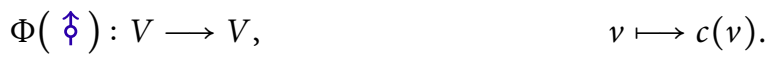

Indeed, a direct check confirms that $\Phi$ respects the defining relations of $\mathcal{O B C}$. Given $\mathrm{a} \in\langle\uparrow, \downarrow\rangle$, we write $V^{\mathrm{a}}:=\Phi(\mathrm{a})$. For example, $V^{\uparrow \downarrow \uparrow}=V \otimes V^{*} \otimes V$.

Theorem 4.1 If the characteristic of the ground field $\mathbb{k}$ is zero, then $\Phi$ is full.

Proof We are required to show that

$$
\Phi: \operatorname{Hom}_{\mathcal{O B C}}(\mathrm{a}, \mathrm{b}) \longrightarrow \operatorname{Hom}_{U(\mathfrak{q})}\left(V^{\mathrm{a}}, V^{\mathrm{b}}\right)
$$

is surjective for all $\mathrm{a}, \mathrm{b} \in\langle\uparrow, \downarrow\rangle$. Suppose a (resp. b) consists of $r_{1}$ (resp. $r_{1}^{\prime}$ ) $\uparrow$ 's and $r_{2}$ (resp. $r_{2}^{\prime}$ ) $\downarrow^{\prime}$ s. Acting by the central element $\sum_{i=1}^{n} e_{i, i}^{\overline{0}}$, one sees that $\operatorname{Hom}_{U(\mathfrak{q})}\left(V^{\mathrm{a}}, V^{\mathrm{b}}\right)=0$ unless $r_{1}+r_{2}^{\prime}=r_{1}^{\prime}+r_{2}$. 
In the nontrivial case, set $r=r_{1}+r_{2}^{\prime}=r_{1}^{\prime}+r_{2}$ and consider the following:

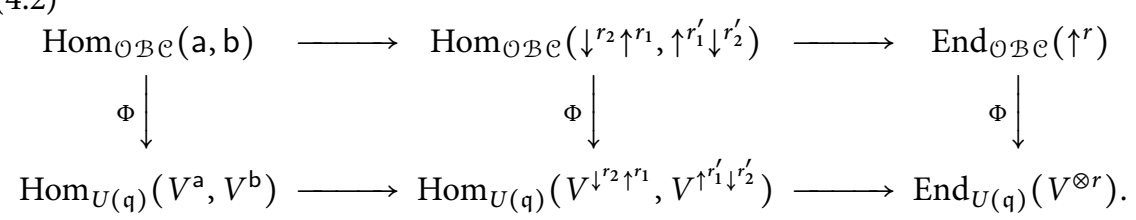

The horizontal maps are all isomorphisms of superspaces. Indeed, the left horizontal maps are given by the symmetric braidings on $\mathcal{O B C}$ and $U(\mathfrak{q})$-smod. The right horizontal maps are the $\mathbb{k}$-linear isomorphisms that hold in any monoidal supercategory with duals. In particular, the top right horizontal map is the $\mathbb{k}$-linear isomorphism given on diagrams by

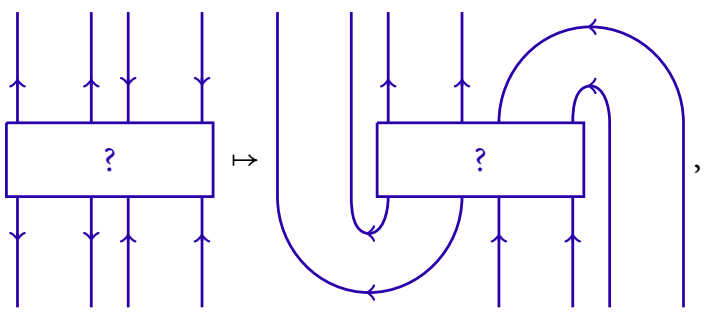

with inverse mapping

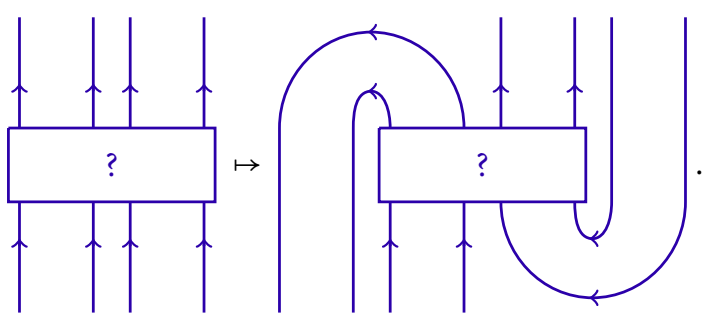

Since the monoidal superfunctor $\Phi$ respects the symmetric braidings and duality, the diagram given in (4.2) commutes. Thus, surjectivity of (4.1) follows from the surjectivity of the right vertical map in (4.2). However, composing the right vertical map in (4.2) with the isomorphism $\varphi$ from Corollary 3.5 gives the superalgebra map $\operatorname{Ser}_{r} \rightarrow \operatorname{End}_{U(\mathfrak{q})}\left(V^{\otimes r}\right)$ from Schur-Weyl-Sergeev duality. When $\mathbb{k}$ has characteristic zero this is known to be surjective by [Ser2] (see also [CW, Section 3.4]).

Remark 4.2 When $\mathbb{k}$ has characteristic zero, Schur-Weyl-Sergeev duality also implies the right vertical map in (4.2) is injective whenever $r \leq n$. It follows that (4.1) is an isomorphism whenever the average length of the words a and b is less than or equal to $n$. In particular, $\Phi$ prescribes an isomorphism of superalgebras $\operatorname{End}_{\mathcal{O B C}}(a) \cong \operatorname{End}_{U(\mathfrak{q})}\left(V^{\mathrm{a}}\right)$ whenever the length of a is less than or equal to $n$. Coupled with Corollary 3.6, this recovers [JK, Theorem 3.5].

Remark 4.3 If $\mathbb{k}$ has positive characteristic, then one can replace $U(\mathfrak{q})$ with the superalgebra of distributions for the supergroup $Q(n)$ and again have the superfunctor $\Phi$. Moreover, the above argument for the fullness of $\Phi$ goes through modulo the 
statement that the map $\operatorname{Ser}_{r} \rightarrow \operatorname{End}_{U(\mathfrak{q})}\left(V^{\otimes r}\right)$ from Schur-Weyl-Sergeev duality is surjective. For given $r$, it can be deduced from [BK2] that this map is surjective whenever $n \geq r$ or the characteristic of $\mathbb{k}$ is greater than $r$. As far as the authors are aware, surjectivity is not known in general. It is reasonable to expect it to hold (and, hence, the fullness of $\Phi)$ under mild conditions on $\mathbb{k}(c f$. [BD1]). Similar remarks apply to injectivity.

\subsection{The Monoidal Superfunctor $\Psi: \mathcal{A O B} \mathcal{B} \rightarrow \mathcal{E} n d(U(\mathfrak{q})$-smod)}

Let $U(\mathfrak{g l})$ (resp. $U(\mathfrak{q})$ ) denote the enveloping superalgebra of $\mathfrak{g l}(V)$ (resp. $\mathfrak{q}(V)$ ). Using the bases given in Section 4.1, we can naturally view $U(\mathfrak{q})$ as Hopf subsuperalgebra of $U(\mathfrak{g l})$.

Let $\Omega \in U(\mathfrak{g l}) \otimes U(\mathfrak{q})$ be the Casimir element given by

$$
\Omega=\sum_{1 \leq i, j \leq n} \bar{e}_{i, j}^{\overline{0}} \otimes e_{j, i}^{\overline{0}}+\bar{e}_{i, j}^{\overline{1}} \otimes e_{j, i}^{\overline{1}}
$$

Given a $U(\mathfrak{g l})$-supermodule $W$ and a $U(\mathfrak{q})$-supermodule $M$, we have an even linear map $W \otimes M \rightarrow W \otimes M$ given by

$$
\Omega .(w \otimes m)=\sum_{1 \leq i, j \leq n} \bar{e}_{i, j}^{\overline{0}} \cdot w \otimes e_{j, i}^{\overline{0}} \cdot m+(-1)^{|w|} \bar{e}_{i, j}^{\overline{1}} \cdot w \otimes e_{j, i}^{\overline{1}} \cdot m,
$$

for all homogeneous $w \in W$ and $m \in M$. By restriction, $W$ is a $U(\mathfrak{q})$-supermodule and so $W \otimes M$ is a $U(\mathfrak{q})$-supermodule via its coproduct. The action of $\Omega$ defines an even $U(\mathfrak{q})$-supermodule homomorphism by [HKS, Theorem 7.4.1] (there it is assumed that $\mathbb{k}=\mathbb{C}$ but the calculations do not depend on this fact). Alternatively, one can use the odd invariant bilinear form given by the supertrace on $\mathfrak{q}$ to define the so-called odd Casimir element of $U(\mathfrak{q}) \otimes U(\mathfrak{q})$, which, by standard arguments, commutes with the image of the coproduct. In turn, since $\Omega$ equals the product of the odd Casimir with $\sqrt{-1} c \otimes 1$, it necessarily defines a supermodule homomorphism; see [BD2, proof of Lemma 3.1] for details.

Theorem 4.4 There is a monoidal superfunctor $\Psi: \mathcal{A O B C} \rightarrow \mathcal{E}$ d $(U(\mathfrak{q})$-smod) by mapping the objects $\uparrow, \downarrow$ to the endofunctors $V \otimes-, V^{*} \otimes-$, respectively, and on morphisms by

$$
\begin{aligned}
& \Psi(\smile): \mathrm{Id} \longrightarrow V \otimes V^{*} \otimes-, \quad \quad m \longmapsto \sum_{i \in I} v_{i} \otimes v_{i}^{*} \otimes m, \\
& \Psi(\curvearrowleft): V^{*} \otimes V \otimes-\longrightarrow \mathrm{Id}, \quad f \otimes v \otimes m \mapsto f(v) m, \\
& \Psi(\widehat{\gamma}): V \otimes V \otimes-\longrightarrow V \otimes V \otimes-, \quad u \otimes v \otimes m \longmapsto(-1)^{|u||v|} v \otimes u \otimes m, \\
& \Psi(\hat{\phi}): V \otimes-\longrightarrow V \otimes-, \quad v \otimes m \longmapsto \Omega(v \otimes m), \\
& \Psi(\hat{\dagger}): V \otimes-\longrightarrow V \otimes-, \quad v \otimes m \longmapsto c(v) \otimes m .
\end{aligned}
$$

Proof To show the existence of the superfunctor requires that we verify the defining relations of $\mathcal{A O B C}$. The first three supernatural transformations are given by maps that are the coevaluation, evaluation, and braiding, respectively, in the supercategory $\mathfrak{s v e c}$. From this it follows that (3.1) and (3.2) are satisfied. A direct calculation verifies 
that (3.6) is also satisfied. The relations in (3.17) follow from the verification of [HKS, (3.1.4) and (3.1.5)], keeping in mind the authors chose to assume that Clifford elements square to minus one and to tensor by $V$ on the right. Our different choices impact the signs that appear in formulas but otherwise have no effect.

\subsection{Bubbles and Central Elements of $U(\mathfrak{q})$}

As is well known (e.g., [CM, Proposition 46]), the supernatural transformations from the identity superfunctor to itself identify as a superalgebra with $Z(\mathfrak{q})$, the supercenter of $U(\mathfrak{q})$. In particular, using the notation for supernatural transformations set in Section $2.2, \Psi\left(\Delta_{k}\right)_{U(\mathfrak{q})}: U(\mathfrak{q}) \rightarrow U(\mathfrak{q})$ is a supermodule homomorphism and $z_{k}:=$ $\Psi\left(\Delta_{k}\right)_{U(\mathfrak{q})}(1)$ lies in $Z(\mathfrak{q})$. In this section we compute these central elements.

To do so requires further notation. For any $k \geq 1$ and $\varepsilon=\left(\varepsilon_{k}, \ldots, \varepsilon_{1}\right) \in \mathbb{Z}_{2}^{k}$, define $|\varepsilon|=\varepsilon_{k}+\cdots+\varepsilon_{1}$. Furthermore, define $\operatorname{sgn}(\varepsilon)= \pm 1$ recursively by

$$
\operatorname{sgn}\left(\varepsilon_{k}, \ldots, \varepsilon_{1}\right)= \begin{cases}(-1)^{\left(\varepsilon_{k}+\overline{1}\right)\left|\left(\varepsilon_{k-1}, \ldots, \varepsilon_{1}\right)\right|} \operatorname{sgn}\left(\varepsilon_{k-1}, \ldots, \varepsilon_{1}\right) & \text { if } k>1, \\ 1 & \text { if } k=1 .\end{cases}
$$

Theorem 4.5 Let $k$ be a positive odd integer; then the central element of $U(\mathfrak{q})$ determined by the even supernatural transformation $\Psi\left(\Delta_{k}\right)$ is

$$
z_{k}=2 \sum_{\substack{\left(i_{k}, \ldots, i_{1}\right) \in \in_{0}^{k}, \varepsilon=\left(\varepsilon_{k}, \ldots, \varepsilon_{1}\right) \in \mathbb{Z}_{2}^{k}, \\ \text { with }|\varepsilon|=\overline{0}}} \operatorname{sgn}(\varepsilon) e_{i_{k-1}, i_{k}}^{\varepsilon_{k}} e_{i_{k-2}, i_{k-1}}^{\varepsilon_{k-1}} \cdots e_{i_{2}, i_{3}}^{\varepsilon_{3}} e_{i_{1}, i_{2}}^{\varepsilon_{2}} e_{i_{k}, i_{1}}^{\varepsilon_{1}} .
$$

Proof We compute:

$$
\Psi\left(\Delta_{k}\right)_{U(\mathfrak{q})}(1)=\left(\mathrm{ev}_{V} \otimes 1\right) \circ\left(1 \otimes \Omega^{k}\right) \circ\left(\gamma_{V, V^{*}} \otimes 1\right) \circ\left(\operatorname{coev}_{V} \otimes 1\right)(1) .
$$

First observe that

(4.4) $\left(\gamma_{V, V^{*}} \otimes 1\right) \circ\left(\operatorname{coev}_{V} \otimes 1\right)(1)=\sum_{i \in I}(-1)^{\left|v_{i}\right|} v_{i}^{*} \otimes v_{i} \otimes 1=\left(1-c_{2,1}\right)\left(\sum_{i_{0} \in I_{0}} v_{i_{0}}^{*} \otimes v_{i_{0}} \otimes 1\right)$,

where $c_{2,1}:=\Psi(\text { q } \$)_{U(\mathfrak{q})} \in \operatorname{End}_{U(\mathfrak{q})}\left(V^{*} \otimes V \otimes U(\mathfrak{q})\right)$.

To continue, it is helpful introduce some notation to simplify formulas. Given $\mathbf{i}=\left(i_{k}, \ldots, i_{0}\right) \in I_{0}^{k+1}$ and $\varepsilon=\left(\varepsilon_{k}, \ldots, \varepsilon_{1}\right) \in \mathbb{Z}_{2}^{k}$, for short let $e_{\mathbf{i}}^{\varepsilon} \in U(\mathfrak{q})$ be given by

$$
e_{\dot{i}}^{\varepsilon}=e_{i_{k-1}, i_{k}}^{\varepsilon_{k}} e_{i_{k-2}, i_{k-1}}^{\varepsilon_{k-1}} \cdots e_{i_{1}, i_{2}}^{\varepsilon_{2}} e_{i_{0}, i_{1}}^{\varepsilon_{1}} .
$$

Given $\varepsilon \in \mathbb{Z}_{2}^{k}$ and $i \in I_{0}$, we write

$$
v_{i}^{|\varepsilon|}= \begin{cases}v_{i} & \text { if }|\varepsilon|=\overline{0} \\ v_{\bar{i}} & \text { if }|\varepsilon|=\overline{1} .\end{cases}
$$

A straightforward induction on $k \geq 1$ proves that for each fixed $i_{0} \in I_{0}$,

$$
(1 \otimes \Omega)^{k} \cdot v_{i_{0}}^{*} \otimes v_{i_{0}} \otimes 1=\sum \operatorname{sgn}(\varepsilon) v_{i_{0}}^{*} \otimes v_{i_{k}}^{|\varepsilon|} \otimes e_{\mathbf{i}}^{\varepsilon},
$$

where the sum is over all $\varepsilon=\left(\varepsilon_{k}, \ldots, \varepsilon_{1}\right) \in \mathbb{Z}_{2}^{k}$ and $\mathbf{i}=\left(i_{k}, \ldots, i_{1}, i_{0}\right) \in I_{0}^{k+1}$. Combining this formula with (4.4) and using $(1 \otimes \Omega) \circ c_{2,1}=-c_{2,1} \circ(1 \otimes \Omega)$ and 


$$
\begin{aligned}
\left(\mathrm{ev}_{V} \otimes 1\right) \circ c_{2,1}=\mathrm{ev}_{V} & \otimes 1 \text { yields } \\
\Psi\left(\Delta_{k}\right)_{U(\mathfrak{q})}(1) & =\left(\mathrm{ev}_{V} \otimes 1\right) \circ\left(1-(-1)^{k} c_{2,1}\right)\left(\sum \operatorname{sgn}(\varepsilon) v_{i_{0}}^{*} \otimes v_{i_{k}}^{|\varepsilon|} \otimes e_{\mathbf{i}}^{\varepsilon}\right) \\
& = \begin{cases}0 & \text { if } k \text { is even, } \\
2 \sum \operatorname{sgn}(\varepsilon) e_{\mathbf{i}}^{\varepsilon} & \text { if } k \text { is odd. }\end{cases}
\end{aligned}
$$

The upper sum is over all $\mathbf{i}=\left(i_{k}, \ldots, i_{1}, i_{0}\right) \in I_{0}^{k+1}$, and $\varepsilon=\left(\varepsilon_{k}, \ldots, \varepsilon_{1}\right) \in \mathbb{Z}_{2}^{k}$. The lower sum is over all $\mathbf{i} \in I_{0}^{k+1}$ with $i_{0}=i_{k}$ and all $\varepsilon \in \mathbb{Z}_{2}^{k}$ with $|\varepsilon|=\overline{0}$. This proves the stated result.

For each integer $t \geq 0$, Sergeev defined an explicit element $S_{t} \in Z(\mathfrak{q})$. As we do not need Sergeev's elements, we do not reproduce their definition here. The interested reader can find it in [Ser1] or [BK3, Section 8]. For completeness' sake we explain how Sergeev's elements relate to those described above. Recall that $\sigma: U(\mathfrak{q}) \rightarrow U(\mathfrak{q})$ is the antipode of $U(\mathfrak{q})$ (see Section 4.1).

Proposition 4.6 For each integer $t \geq 1, z_{2 t-1}=-2 \sigma\left(S_{t}\right)$.

Proof By expanding the recursive formula for $S_{t}$, we see that it is a sum with coefficients of \pm 1 over precisely the same set of monomials as given by the formula for $z_{2 t-1}$ in Theorem 4.5, except that they are in reverse order. That is, $\sigma\left(S_{t}\right)$ and $z_{2 t-1}$ are sums over precisely the same set of monomials. All that remains is to verify that the sign in front of each monomial agrees. This is a straightforward check, keeping in mind that since $\sigma$ is a superalgebra anti-involution, if $x_{1}, \ldots, x_{k} \in \mathfrak{q}$, then $\sigma\left(x_{1} \cdots x_{k}\right)=(-1)^{\delta} x_{k} \cdots x_{1}$, where $\delta=k+\sum_{1 \leq r<s \leq k}\left|x_{r}\right|\left|x_{s}\right|$. It is also helpful in comparing signs to verify that a closed formula for $\operatorname{sgn}\left(\varepsilon_{k}, \ldots, \varepsilon_{1}\right)$ is given by

$$
\operatorname{sgn}\left(\varepsilon_{k}, \ldots, \varepsilon_{1}\right)=(-1)^{\varepsilon_{k-1}+\varepsilon_{k-3}+\cdots+\varepsilon_{p}+\sum_{1 \leq r s \leq k} \varepsilon_{r} \varepsilon_{s}},
$$

where $p=1$ if $k$ is even and $p=2$ if $k$ is odd.

Remark 4.7 Assume $\mathbb{k}$ has characteristic zero. By our basis theorem for $\mathcal{A O B} \mathcal{B}$, the set $\left\{\Delta_{1}, \Delta_{3}, \Delta_{5}, \ldots\right\}$ is algebraically independent (see also Remark 5.5). Moreover, Sergeev's elements are known to generate $Z(\mathfrak{q})$ for every $n \geq 1$ by [Serl]. Therefore, $\Psi$ defines a surjective homomorphism $\operatorname{End}_{\mathcal{A O B} \mathcal{B}}(\mathbb{1}) \rightarrow \operatorname{End}_{\mathcal{E} n d(U(\mathfrak{q}) \text {-smod) }}(\mathrm{Id})$.

\section{The Generic Verma Supermodule}

We now introduce the generic Verma supermodule. This supermodule will play a key role in proving the basis theorem for $\mathcal{A O B C}$. Analogous modules exist for Lie (super)algebras of other types. Our approach could be used to give a different proof of the basis theorem for $\mathcal{A O B}$ in type $\mathrm{A}$ and to obtain similar results in other types (e.g., see $[\mathrm{BDE}+])$.

\subsection{The Polynomial Ring}

Let $U\left(\mathfrak{h}_{\overline{0}}\right)$ denote the subsuperalgebra of $U(\mathfrak{h})$ generated by $\left\{h_{i} \mid i=1, \ldots, n\right\}$, where for brevity we set $h_{i}=e_{i, i}^{\overline{0}}$ for $i=1, \ldots, n$. We put the usual $\mathbb{Z}$-grading on $U\left(\mathfrak{h}_{\overline{0}}\right)$ by 
putting each $h_{i}$ in degree 1 . Since $\mathfrak{h}_{\overline{0}}$ is an abelian Lie superalgebra concentrated in parity zero, $U\left(\mathfrak{h}_{\overline{0}}\right)$ is nothing more than a polynomial ring on generators $h_{n}, \ldots, h_{1}$. We refine the $\mathbb{Z}$-grading on $U\left(\mathfrak{h}_{\overline{0}}\right)$ by also putting a graded lexicographic order on the monomials with the convention that $h_{n}>\cdots>h_{1}$; that is, $h_{n}^{r_{n}} \cdots h_{1}^{r_{1}}<h_{n}^{s_{n}} \cdots h_{1}^{s_{1}}$ if and only if either $\sum_{i} r_{i}<\sum_{i} s_{i}$ or $\sum_{i} r_{i}=\sum_{i} s_{i}$ and if $t=1, \ldots, n$ is maximal with $r_{t} \neq s_{t}$, then $r_{t}<s_{t}$.

\subsection{The Generic Verma Supermodule}

Let $U(\mathfrak{b})$ denote the subsuperalgebra of $U(\mathfrak{q})$ generated by $U(\mathfrak{h})$ and $U(\mathfrak{n})$. By the PBW theorem, we have $U(\mathfrak{b})=U(\mathfrak{h}) \otimes U(\mathfrak{n})$ as superspaces. Since the span of the monomials of positive degree, $U(\mathfrak{n})_{+}$, is an ideal of $U(\mathfrak{b})$ with $U(\mathfrak{b}) / U(\mathfrak{n})_{+} \cong U(\mathfrak{h})$, we can and will view $U(\mathfrak{h})$ as a $U(\mathfrak{b})$-supermodule by inflation. Define the generic Verma supermodule to be the $U(\mathfrak{q})$-supermodule

$$
M=U(\mathfrak{q}) \otimes_{U(\mathfrak{b})} U(\mathfrak{h}) \cong U(\mathfrak{q}) \otimes_{U(\mathfrak{b})}\left(U(\mathfrak{h}) \otimes_{U\left(\mathfrak{h}_{\overline{0}}\right)} U\left(\mathfrak{h}_{\overline{0}}\right)\right) .
$$

We will write $\widehat{u}:=1 \otimes 1 \otimes 1 \in M$ for the "highest weight vector". While we do not need this fact, note that if $W$ is a $U(\mathfrak{q})$-supermodule and $w \in W$ is a homogeneous weight vector for $U\left(\mathfrak{h}_{\overline{0}}\right)$ such that $e_{i, j}^{\varepsilon} w=0$ for all $1 \leq i<j \leq n$ and all $\varepsilon \in \mathbb{Z}_{2}$, then there is a unique $U(\mathfrak{q})$-supermodule homomorphism $M \rightarrow W$ such that $\widehat{u} \mapsto w$.

For brevity, set $N=n(n-1) / 2$ and let $\left\{f_{1}, \ldots, f_{N}\right\}$ (resp. $\left\{\bar{f}_{1}, \ldots, \bar{f}_{N}\right\}$ ) be any basis for $\mathfrak{n}_{\overline{0}}^{-}\left(\right.$resp. $\left.\mathfrak{n}_{\overline{1}}^{-}\right)$such that

$$
\left\{e_{i, j}^{\overline{0}} \mid 1 \leq j<i \leq n\right\}=\left\{f_{1}, \ldots, f_{N}\right\} \quad \text { and } \quad\left\{e_{i, j}^{\overline{1}} \mid 1 \leq j<i \leq n\right\}=\left\{\bar{f}_{1}, \ldots, \bar{f}_{N}\right\} .
$$

In particular, a PBW basis for $U\left(\mathfrak{n}^{-}\right)$is given by the monomials $f_{1}^{a_{1}} \cdots f_{N}^{a_{N}} \bar{f}_{1}^{b_{1}} \cdots \bar{f}_{N}^{b_{N}}$, where $a_{1}, \ldots, a_{N} \in \mathbb{Z}_{\geq 0}$ and $b_{1}, \ldots, b_{N} \in\{0,1\}$. By the PBW theorem $M$ is a free right $U\left(\mathfrak{h}_{\overline{0}}\right)$-supermodule on basis

$$
f_{1}^{a_{1}} \cdots f_{N}^{a_{N}} \bar{f}_{1}^{b_{1}} \cdots \bar{f}_{N}^{b_{N}} \otimes \bar{h}_{1}^{c_{1}} \cdots \bar{h}_{n}^{c_{n}} \otimes 1
$$

where each $a_{k} \in \mathbb{Z}_{\geq 0}$ and $b_{k}, c_{k} \in\{0,1\}$. In particular, the superspace $M$ inherits a $\mathbb{Z}$-grading (hence a $\mathbb{Z}$-filtration) from the right action of $U\left(\mathfrak{h}_{\overline{0}}\right)$ where for $t \in \mathbb{Z}, M_{t}$ is the span of

$$
f_{1}^{a_{1}} \cdots f_{N}^{a_{N}} \bar{f}_{1}^{b_{1}} \cdots \bar{f}_{N}^{b_{N}} \otimes \bar{h}_{1}^{c_{1}} \cdots \bar{h}_{n}^{c_{n}} \otimes U\left(\mathfrak{h}_{\overline{0}}\right)_{t}
$$

for all $a_{k} \in \mathbb{Z}_{\geq 0}$ and $b_{k}, c_{k} \in\{0,1\}$. More generally, for any a $\in\langle\uparrow, \downarrow\rangle$, the superspace $V^{\mathrm{a}} \otimes M$ is a free right $U\left(\mathfrak{h}_{\overline{0}}\right)$-supermodule and is similarly a graded (hence filtered) superspace.

Lemma 5.1 Suppose $1 \leq i, j \leq n$, and $\varepsilon \in \mathbb{Z}_{2}$. Left multiplication by $e_{i, j}^{\varepsilon}$ prescribes a map $M \rightarrow M$ that is

(i) homogeneous degree 0 when $i>j$,

(ii) filtered degree 1 when $i \leq j$.

Proof Since $M$ is a $\left(U(\mathfrak{q}), U\left(\mathfrak{h}_{\overline{0}}\right)\right)$-bisupermodule with the grading coming from the right action by $U\left(\mathfrak{h}_{0}\right)$, it suffices to show that the result of acting on (5.1) on the left by $e_{i, j}^{\varepsilon}$ is (i) in $M_{0}$ when $i>j$, and (ii) in $M_{\leq 1}$ when $i \leq j$. 
Part (i) follows from the fact that $e_{i, j}^{\varepsilon}$ is an element of the subsuperalgebra $U\left(\mathfrak{n}^{-}\right)$ whenever $i>j$. That is, since monomials of the form $f_{1}^{a_{1}} \cdots f_{N}^{a_{N}} \bar{f}_{1} b_{1} \cdots \bar{f}_{N}{ }^{b_{N}}$ are a basis for $U\left(\mathfrak{n}^{-}\right)$by the PBW theorem, after acting by $e_{i, j}^{\varepsilon}$ in $U\left(\mathfrak{n}^{-}\right)$one can rewrite the result as a sum of elements that lie in $M_{0}$.

For part (ii) one can argue by induction on

$$
a_{1}+b_{1}+\cdots+a_{N}+b_{N}
$$

Namely, act by $e_{i, j}^{\varepsilon}$ on (5.1) and then use the commutator formulas $U(\mathfrak{q})$ given in [DW, Sections 2 and 3] to rewrite the expression into a sum of terms with smaller (5.2). The base case when this sum equals zero also follows by a calculation using the commutator formulas of [DW].

Lemma 5.2 For any $\mathrm{a} \in\langle\uparrow, \downarrow\rangle$, the map $V \otimes\left(V^{\mathrm{a}} \otimes M\right) \rightarrow V \otimes\left(V^{\mathrm{a}} \otimes M\right)$ given by the action of $\Omega$ is filtered degree 1 .

Proof This follows from the definition of the Casimir (4.3) and Lemma 5.1.

Using the previous lemma we can show that the composition of $\Psi$ followed by evaluation at $M$ defines a filtered monoidal superfunctor $\Psi_{M}: \mathcal{A O B C} \rightarrow \mathfrak{f} \mathfrak{s} \mathfrak{v e c}$. Indeed, it is straightforward to see that $\Psi_{M}(d)$ is filtered degree 0 whenever $d$ is an oriented Brauer-Clifford diagram. The fact that $\Psi_{M}(d)$ is a filtered map with filtered degree $\operatorname{deg}(d)$ whenever $d$ is a dotted oriented Brauer-Clifford diagram with bubbles follows from Lemma 5.2.

\subsection{Some $\Psi_{M}$ Calculations}

In this subsection we prove several lemmas concerning the superfunctor $\Psi_{M}$, which will be used in Section 6 to prove our basis theorem for $\mathcal{A O B C}$.

Lemma 5.3 Whenever $1 \leq i \leq n$, the degree 1 components of

$$
\Psi_{M}(\hat{\boldsymbol{\phi}})\left(v_{i} \otimes \widehat{u}\right) \quad \text { and } \quad \Psi_{M}(\hat{\boldsymbol{\phi}})\left(v_{\bar{i}} \otimes \widehat{u}\right)
$$

are $v_{i} \otimes \widehat{u} h_{i}$ and $-v_{\bar{i}} \otimes \widehat{u} h_{i}$, respectively.

Proof Note that $e_{i, j}^{\varepsilon} \widehat{u}$ has degree 0 unless $\varepsilon=0$ and $i=j$, in which case we have $e_{i, j}^{\varepsilon} \widehat{u}=h_{i} \widehat{u}=\widehat{u} h_{i}$. Thus, using (4.3) we see the degree 1 components of

$$
\Psi_{M}(\hat{\boldsymbol{\phi}})\left(v_{i} \otimes \widehat{u}\right) \quad \text { and } \quad \Psi_{M}(\hat{\boldsymbol{\phi}})\left(v_{\bar{i}} \otimes \widehat{u}\right)
$$

are as given by the lemma.

Lemma 5.4 The degree $k$ component of $\Psi_{M}\left(\Delta_{k}\right)(\widehat{u})$ is $2 \widehat{u}\left(h_{n}^{k}+\cdots+h_{1}^{k}\right)$ whenever $k$ is odd. 
Proof In the following computation of $\Psi_{M}\left(\Delta_{k}\right)(\widehat{u})$ we list only the top degree terms:

$$
\begin{aligned}
& \widehat{u} \longmapsto \sum_{1 \leq i \leq n} v_{i} \otimes v_{i}^{*} \otimes \widehat{u}+v_{\bar{i}} \otimes v_{\bar{i}}^{*} \otimes \widehat{u} \\
& \longmapsto \sum_{1 \leq i \leq n} v_{i}^{*} \otimes v_{i} \otimes \widehat{u}-v_{\bar{i}}^{*} \otimes v_{\bar{i}} \otimes \widehat{u} \\
& \longmapsto \sum_{1 \leq i \leq n} v_{i}^{*} \otimes v_{i} \otimes \widehat{u} h_{i}^{k}-(-1)^{k} v_{\bar{i}}^{*} \otimes v_{\bar{i}} \otimes \widehat{u} h_{i}^{k}+\cdots \\
& \longmapsto \sum_{1 \leq i \leq n} \widehat{u} h_{i}^{k}-(-1)^{k} \widehat{u} h_{i}^{k}+\cdots \\
& \text { (apply } \Psi_{M}(\smile) \text { ) } \\
& \text { (apply } \left.\Psi_{M}(\Varangle)\right) \\
& \text { (apply } \Psi_{M}(\downarrow \hat{\phi} k) \text { and } \\
& \text { use Lemma 5.3) } \\
& \text { (apply } \Psi_{M}(\curvearrowleft) \text { ). }
\end{aligned}
$$

Now, assume $k$ is odd to get $\Psi_{M}\left(\Delta_{k}\right)(\widehat{u})=2 \widehat{u}\left(h_{n}^{k}+\cdots+h_{1}^{k}\right)+\cdots$.

Remark 5.5 Assume $\mathbb{k}$ has characteristic zero. By taking $n$ sufficiently large, the previous lemma along with the fact the first $n$ power sums in $h_{n}, \ldots, h_{1}$ are algebraically independent can be used to show $\Delta_{1}, \Delta_{3}, \Delta_{5}, \ldots$ are algebraically independent.

The next two lemmas concern $\Psi_{M}(d)$ for certain diagrams of the form $d: \uparrow^{r} \rightarrow$ $\uparrow^{r}$. It will be convenient to let $x_{k}: \uparrow^{r} \rightarrow \uparrow^{r}$ denote the diagram obtained from the identity diagram by placing a single $\bullet$ on the $k$-th strand from the right. We will also let $(i, j): \uparrow^{r} \rightarrow \uparrow^{r}$ denote the crossing of the $i$-th and $j$-th strands whenever $1 \leq i, j \leq r$. For example, if $r=8$, then

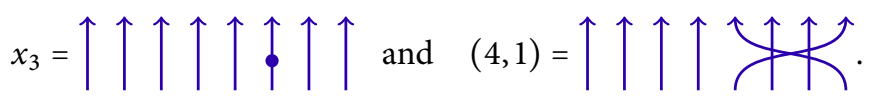

Lemma 5.6 The degree 1 component of $\Psi_{M}\left(x_{k}\right)\left(v_{r} \otimes \cdots v_{1} \otimes \widehat{u}\right)$ is $v_{r} \otimes \cdots v_{1} \otimes \widehat{u} h_{k}$.

Proof First, $x_{k}-(k, 1) x_{1}(k, 1)$ is a linear combination of oriented Brauer-Clifford diagrams whenever $k>1$. Hence, since $\Psi_{M}$ is filtered and oriented Brauer-Clifford diagrams are degree 0 , the degree 1 components of $\Psi_{M}\left(x_{k}\right)\left(v_{r} \otimes \cdots \otimes v_{1} \otimes \widehat{u}\right)$ and $\Psi_{M}\left((k, 1) x_{1}(k, 1)\right)\left(v_{r} \otimes \cdots \otimes v_{1} \otimes \widehat{u}\right)$ are equal. Thus, it suffices to prove the case $k=1$, which follows from Lemma 5.3.

Let us fix the following notation for the remainder of the paper. Given a dotted oriented Brauer-Clifford diagram $d$, let undot $(d)$ denote the oriented Brauer-Clifford diagram obtained from $d$ by removing all $\bullet$ 's. For example, if

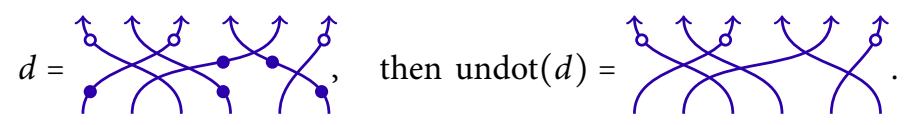

Now, suppose $d: \uparrow^{r} \rightarrow \uparrow^{r}$ is a normally ordered dotted oriented Brauer-Clifford diagram (as in Definition 3.8) without bubbles. We let $\beta_{k}(d)$ denote the number of $\bullet$ 's on the $k$-th strand of $d$, where we count strands right-to-left according to their position on the bottom boundary of $d$. In particular, we have

$$
d=\operatorname{undot}(d) \circ x_{r}^{\beta_{r}(d)} \circ \cdots \circ x_{1}^{\beta_{1}(d)} .
$$


Finally, given $g \in \operatorname{End}_{\mathcal{O B}}\left(\uparrow^{r}\right)$ we let $v(g) \in V^{\otimes r}$ denote the image of $v_{r} \otimes \cdots \otimes v_{1}$ under $\Phi(g)$. For example, when $d$ as in (5.3) we have

$$
v(\operatorname{undot}(d))=-\sqrt{-1} v_{\overline{4}} \otimes v_{3} \otimes v_{\overline{6}} \otimes v_{1} \otimes v_{5} \otimes v_{\overline{2}} .
$$

With this notation in mind, the following result follows immediately from Lemma 5.6.

Lemma 5.7 For any normally ordered dotted oriented Brauer-Clifford diagram $d: \uparrow^{r} \rightarrow \uparrow^{r}$ without bubbles, the top degree component of $\Psi_{M}(d)\left(v_{r} \otimes \cdots v_{1} \otimes \widehat{u}\right)$ is $v(\operatorname{undot}(d)) \otimes \widehat{u} h_{r}^{\beta_{r}(d)} \ldots h_{1}^{\beta_{1}(d)}$.

\section{Proof of the Main Result}

We can now prove the key special case of the main result. Namely, the normally ordered dotted oriented Brauer-Clifford diagrams with bubbles provide a basis for $\operatorname{End}_{\mathcal{A O B} \mathcal{C}}\left(\uparrow^{r}\right)$.

Theorem 6.1 Assume that $\mathbb{k}$ has characteristic zero. Then the set of equivalence classes of normally ordered dotted oriented Brauer-Clifford diagrams with bubbles of type $\uparrow^{r} \rightarrow$ $\uparrow^{r}$ form a basis for $\operatorname{End}_{\mathcal{A O B}}\left(\uparrow^{r}\right)$.

Proof By Lemma 3.13, the proposed basis spans $\operatorname{End}_{\mathcal{A O B} \mathcal{B}}\left(\uparrow^{r}\right)$. Toward showing linear independence, note that any linear combination of normally ordered dotted oriented Brauer-Clifford diagrams with bubbles of type $\uparrow^{r} \rightarrow \uparrow^{r}$ can be written in the form

$$
\sum_{d} f_{d}\left(\Delta_{1}, \Delta_{3}, \Delta_{5}, \ldots\right) d
$$

where the sum is over all normally ordered dotted oriented Brauer-Clifford diagrams $d: \uparrow^{r} \rightarrow \uparrow^{r}$ (without bubbles), and where the $f_{d}$ 's are polynomials in countably many variables, only finitely many of which are nonzero. Set $B=\left\{d \mid f_{d} \neq 0\right\}$.

We will show (6.1) is nonzero whenever $B \neq \varnothing$ (completing the proof of the theorem) by showing its image under $\Psi_{M}$ is nonzero whenever $n$ is sufficiently large. In turn, this will follow from the fact that, when we choose $n$ sufficiently large to ensure the relevant power sums are algebraically independent for any $d \in B$ :

$$
f_{d}\left(h_{n}+\cdots+h_{1}, h_{n}^{3}+\cdots+h_{1}^{3}, h_{n}^{5}+\cdots+h_{1}^{5}, \ldots\right) \neq 0 .
$$

Recall from Section 5.1 that we have a graded lexicographic ordering on the monomials of $U(\mathfrak{h})$. For the rest of the proof we assume that $n$ is large enough so that for each $d \in B$, the leading monomial of the symmetric polynomial (6.2) with respect to this ordering does not contain any of $h_{r}, \ldots, h_{1}$.

Given $d \in B$, it follows from Lemma 5.4 that the top degree component of

$$
\Psi_{M}\left(f_{d}\left(\Delta_{1}, \Delta_{3}, \ldots\right)\right)\left(v_{r} \otimes \cdots \otimes v_{1} \otimes \widehat{u}\right)
$$

is of the form $v_{r} \otimes \cdots \otimes v_{1} \otimes \widehat{u} g_{d}\left(h_{n}, \ldots, h_{1}\right)$ where $g_{d}\left(h_{n}, \ldots, h_{1}\right)$ is some homogeneous symmetric polynomial. Fix $d_{0} \in B$ with

$$
\operatorname{deg} d_{0}+\operatorname{deg} g_{d_{0}} \geq \operatorname{deg} d+\operatorname{deg} g_{d} \text { for all } d \in B .
$$


Set

$$
B_{0}=\left\{d \in B \mid \operatorname{undot}(d)=\operatorname{undot}\left(d_{0}\right) \text { and } \operatorname{deg} d_{0}+\operatorname{deg} g_{d_{0}}=\operatorname{deg} d+\operatorname{deg} g_{d}\right\} .
$$

It follows from (6.3) and Lemma 5.7 that the top degree component of the image of $v_{r} \otimes \cdots \otimes v_{1} \otimes \widehat{u}$ under $\Psi_{M}\left(\sum_{d} f_{d}\left(\Delta_{1}, \Delta_{3}, \Delta_{5}, \ldots\right) d\right)$ is of the form

$$
\sum_{d \in B_{0}} v\left(\operatorname{undot}\left(d_{0}\right)\right) \otimes \widehat{u} g_{d}\left(h_{n}, \ldots, h_{1}\right) h_{r}^{\beta_{r}(d)} \cdots h_{1}^{\beta_{1}(d)}+w,
$$

where $w$ lies in the $U(\mathfrak{h})$-span of the basis elements of the form $v(d) \otimes u$ with $v(d) \neq v\left(\right.$ undot $\left.\left(d_{0}\right)\right)$. Recall that since $n$ was chosen sufficiently large, the elements $h_{r}, \ldots, h_{1}$ do not appear in leading monomials of each $g_{d}\left(h_{n}, \ldots, h_{1}\right)$. Therefore, since a diagram $d \in B_{0}$ is completely determined by $\beta_{1}(d), \ldots, \beta_{r}(d)$, it follows that the leading monomials of $g_{d}\left(h_{n}, \ldots, h_{1}\right) h_{r}^{\beta_{r}(d)} \ldots h_{1}^{\beta_{1}(d)}$ for $d \in B_{0}$ are pairwise distinct. Thus, (6.4) is nonzero, which implies (6.1) is nonzero, as desired.

\subsection{Proof of Theorem 3.9}

It is straightforward to see that the validity of Theorem 3.9 when $\mathbb{k}$ has characteristic zero is equivalent to the following lemma.

Lemma 6.2 Assume $\mathbb{k}$ has characteristic zero. Then for any $\mathrm{a}, \mathrm{b} \in\langle\uparrow, \downarrow\rangle$, the superspace $\operatorname{Hom}_{\mathcal{A O B C}}(\mathrm{a}, \mathrm{b})_{\leq k}$ has basis given by equivalence classes of normally ordered dotted oriented Brauer-Clifford diagrams with bubbles of type $\mathrm{a} \rightarrow \mathrm{b}$ with at most $k \bullet$ 's.

Proof It follows from Lemma 3.13 that the proposed basis spans $\operatorname{Hom}_{\mathcal{A O B} \mathcal{B}}(\mathrm{a}, \mathrm{b})_{\leq k}$. In particular, $\operatorname{Hom}_{\mathcal{A O B} \mathcal{B}}(\mathrm{a}, \mathrm{b})_{\leq k}$ is finite-dimensional over $\mathbb{k}$. Hence, it suffices to show the proposed basis has size $\operatorname{dim}_{\mathbb{k}} \operatorname{Hom}_{\mathcal{A O} \mathcal{B} \mathcal{C}}(\mathrm{a}, \mathrm{b})_{\leq k}$. Now, suppose a (resp. b) consists of $r_{1}$ (resp. $r_{1}^{\prime}$ ) $\uparrow^{\prime}$ s and $r_{2}$ (resp. $r_{2}^{\prime}$ ) $\downarrow^{\prime}$ 's. If $r_{1}+r_{2}^{\prime} \neq r_{1}^{\prime}+r_{2}$, then there are no oriented Brauer-Clifford diagrams of type $a \rightarrow b$, whence $\operatorname{Hom}_{\mathcal{A O B C}}(a, b)=0$. Thus, we can assume that $r_{1}+r_{2}^{\prime}=r_{1}^{\prime}+r_{2}=: r$. In this case we have $\mathbb{k}$-linear isomorphisms

$$
\operatorname{Hom}_{\mathcal{A O B C}}(\mathrm{a}, \mathrm{b})_{\leq k} \longrightarrow \operatorname{Hom}_{\mathcal{A O B} \mathcal{B}}\left(\downarrow^{r_{2}} \uparrow^{r_{1}}, \uparrow^{r_{1}^{\prime}} \downarrow^{r_{2}^{\prime}}\right)_{\leq k} \longrightarrow \operatorname{End}_{\mathcal{A O B C}}\left(\uparrow^{r}\right)_{\leq k}
$$

defined on diagrams in the same manner as the top horizontal maps in (4.2). In particular, $\operatorname{dim}_{\mathbb{k}} \operatorname{Hom}_{\mathcal{A O B} \mathcal{B}}(\mathrm{a}, \mathrm{b})_{\leq k}=\operatorname{dim}_{\mathbb{K}} \operatorname{End}_{\mathcal{A O B \mathcal { C }}}\left(\uparrow^{r}\right)_{\leq k}$. On the other hand, there are precisely $r$ strands in any dotted oriented Brauer-Clifford diagram of type $a \rightarrow b$. It follows that there are the same number of normally ordered dotted oriented BrauerClifford diagrams with bubbles with at most $k \bullet$ 's of type $\mathrm{a} \rightarrow \mathrm{b}$ as there are of type $\uparrow^{r} \rightarrow \uparrow^{r}$. Thus, the result follows from Theorem 6.1.

\subsection{The Positive Characteristic Case}

We now explain how to deduce Theorem 3.9 when $\mathbb{k}$ has positive characteristic and, more generally, is an arbitrary graded commutative $\mathbb{Z}_{2}$-graded ring of characteristic not two. We first observe that the definitions given in Section 2.2 work equally well if $\mathbb{k}$ is replaced with an arbitrary graded commutative $\mathbb{Z}_{2}$-graded ring, $R$, and $\mathbb{k}$-superspaces are replaced with $\mathbb{Z}_{2}$-graded $R$-modules. We refer to these as (monoidal) $R$-supercategories. We define $\mathcal{A} \mathcal{O B} \mathcal{C}_{R}$ to be the monoidal $R$-supercategory given 
by the same generators and relations used in Definition 3.7. For example, we have the integral form $\mathcal{A O B} C_{\mathbb{Z}}$ of the degenerate affine oriented Brauer-Clifford supercategory.

With the above in mind we have the following integral version of Theorem 3.9.

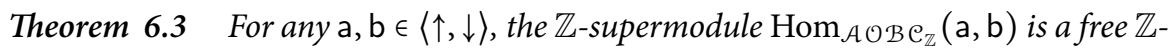
supermodule with basis given by equivalence classes of normally ordered dotted oriented Brauer-Clifford diagrams with bubbles of type $\mathrm{a} \rightarrow \mathrm{b}$.

Proof Since the relations for $\mathcal{A O B C}$ involve only integral coefficients, the arguments given in Section 3.5 apply to $\mathcal{A O B} \mathcal{C}_{\mathbb{Z}}$, and so the normally ordered dotted oriented Brauer-Clifford diagrams with bubbles of type $\mathrm{a} \rightarrow \mathrm{b}$ span $\operatorname{Hom}_{\mathcal{A O} \mathcal{B C}_{\mathbb{Z}}}(\mathrm{a}, \mathrm{b})$ as a $\mathbb{Z}$-supermodule. On the other hand, consider a finite sum

$$
\sum_{d} f_{d} d
$$

where the sum is over normally ordered dotted oriented Brauer-Clifford diagrams with bubbles of type $\mathrm{a} \rightarrow \mathrm{b}$ and where the $f_{d}$ 's are integers. There is an obvious superfunctor $\mathcal{A O B C} \mathcal{C}_{\mathbb{Z}} \rightarrow \mathcal{A O B C} \mathcal{C}_{\mathbb{C}}$ that can be applied to (6.5), and linear independence follows from Theorem 3.9.

Let $\mathbb{k}$ be a graded commutative $\mathbb{Z}_{2}$-graded ring. If $\mathcal{C}_{\mathbb{Z}}$ denotes a (monoidal) $\mathbb{Z}$-supercategory, then by base change one can define a (monoidal) $\mathbb{k}$-supercategory $\mathcal{C}_{\mathbb{Z}} \otimes \mathbb{k}$. Namely, the objects of $\mathcal{C}_{\mathbb{Z}} \otimes \mathbb{k}$ are the objects of $\mathcal{C}_{\mathbb{Z}}$ and the morphisms are

$$
\operatorname{Hom}_{\mathcal{C}_{\mathbb{Z}} \otimes \mathbb{k}}(\mathrm{a}, \mathrm{b})=\operatorname{Hom}_{\mathcal{C}_{\mathbb{Z}}}(\mathrm{a}, \mathrm{b}) \otimes_{\mathbb{Z}} \mathbb{k} .
$$

Composition, the monoidal structure, etc., are extended to $\mathcal{C}_{\mathbb{Z}} \otimes \mathbb{k}$ by linearity. There are obvious mutually inverse superfunctors that provide an isomorphism of monoidal supercategories between $\mathcal{A O B} \mathcal{C}_{\mathbb{Z}} \otimes \mathbb{k}$ and $\mathcal{A O B} \mathcal{C}_{\mathbb{k}}$. The previous theorem and base change immediately implies the following result.

Corollary 6.4 Let $\mathbb{k}$ be an arbitrary graded commutative $\mathbb{Z}_{2}$-graded ring of characteristic not two. For any $\mathrm{a}, \mathrm{b} \in\langle\uparrow, \downarrow\rangle$, the $\mathbb{k}$-supermodule $\operatorname{Hom}_{\mathcal{A O B}} \mathcal{C}_{\mathrm{k}}(\mathrm{a}, \mathrm{b})$ is a free $\mathbb{k}$-supermodule with basis given by equivalence classes of normally ordered dotted oriented Brauer-Clifford diagrams with bubbles of type $\mathrm{a} \rightarrow \mathrm{b}$.

We mention one other application of our basis theorem.

Corollary 6.5 Let $\mathbb{k}$ be a field of characteristic not two. The subsuperalgebra of $\operatorname{End}_{\mathcal{A O B} \mathcal{B}}\left(\uparrow^{r}\right)$ consisting of linear combinations of dotted oriented Brauer-Clifford diagrams without bubbles is isomorphic to the degenerate affine Sergeev superalgebra ${ }^{2}$ $\mathrm{ASerg}_{r}$ introduced in [Naz, Section 3].

Proof The superalgebra ASerg ${ }_{r}$ has a presentation with even generators $s_{1}, \ldots, s_{r-1}$, $x_{1}, \ldots, x_{r}$, and odd generators $c_{1}, \ldots, c_{r}$ subject to the relations (for all admissible $i, j$ ):

(a) $s_{i}^{2}=1, s_{i} s_{j}=s_{j} s_{i}$ when $|i-j|>1, s_{i} s_{i+1} s_{i}=s_{i+1} s_{i} s_{i+1}$;

\footnotetext{
${ }^{2}$ This is also known as the degenerate affine Hecke-Clifford superalgebra.
} 
(b) $c_{i}^{2}=1, c_{i} c_{j}=-c_{j} c_{i}$ when $i \neq j$;

(c) $x_{i} x_{j}=x_{j} x_{i}$

(d) $c_{i} x_{i}=-x_{i} c_{i}, c_{i} x_{j}=x_{j} c_{i}$ when $i \neq j$;

(e) $s_{i} x_{i}=x_{i+1} s_{i}-1-c_{i} c_{i+1}$.

By checking relations, we see that

$$
\left.v\left(s_{i}\right)=\uparrow^{r-i-1} \nmid \uparrow^{i-1}, \quad v\left(c_{i}\right)=\uparrow^{r-i} \uparrow \uparrow^{i-1}, \quad v\left(x_{i}\right)=\uparrow^{r-i} \uparrow\right\rceil^{i-1}
$$

defines a superalgebra homomorphism $v: \operatorname{ASerg}_{r} \rightarrow \operatorname{End}_{\mathcal{A O B \mathcal { B }}}\left(\uparrow^{r}\right)$. Take note that this map follows our convention of numbering strands from right-to-left. The image of this map is the subsuperalgebra of $\operatorname{End}_{\mathcal{A O B C}}\left(\uparrow^{r}\right)$ spanned by the dotted oriented Brauer-Clifford diagrams without bubbles. From Corollary 6.4 and the PBW-type basis for ASerg $r$ given in [Kle, Theorem 14.2.2], one can verify that this map is an isomorphism onto its image.

\section{Cyclotomic Quotients}

Fix $a, b \in \mathbb{Z}_{\geq 0}$ and $m_{i} \in \mathbb{k}$ for each $1 \leq i \leq a$. Let $f(t)=t^{b} \prod_{1 \leq i \leq a}\left(t^{2}-m_{i}\right), \ell=2 a+b$, and $\mathcal{O B C}^{f}$ be as in Section 1.3.

\subsection{Bases for Cyclotomic Quotients}

Since $\mathcal{O B} \mathcal{B}^{f}$ is a quotient of $\mathcal{A O B C}$, we can interpret any dotted oriented BrauerClifford diagram with bubbles as a morphism in $\mathcal{O B} \mathcal{C}^{f}$.

Theorem 7.1 For any $\mathrm{a}, \mathrm{b} \in\langle\uparrow, \downarrow\rangle$, the superspace $\operatorname{Hom}_{\mathcal{O B} \mathcal{B}^{f}}(\mathrm{a}, \mathrm{b})$ has basis given by equivalence classes of normally ordered dotted oriented Brauer-Clifford diagrams with bubbles of type $\mathrm{a} \rightarrow \mathrm{b}$ with fewer than $\ell \bullet$ 's on each strand.

A full proof of this theorem can be found in Section 8. Note that it is easy to show the proposed basis in Theorem 7.1 spans the appropriate Hom-space. Indeed, any dotted oriented Brauer-Clifford diagram with bubbles having $\ell$ 's on one of its strands can be realized as a linear combination of diagrams with fewer total 's by using Proposition 3.10 to slide those $\ell \cdot$ 's to the right side of the picture, and then reducing as prescribed by $f$. For example, the following holds in $\mathcal{O B} \mathcal{C}^{f}$ when $f(t)=t$ :

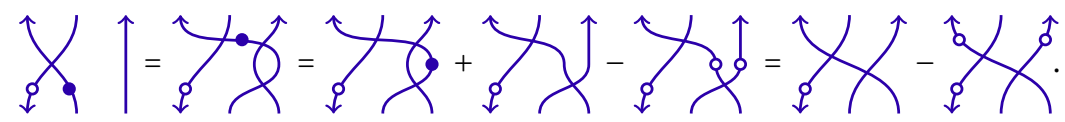

\subsection{Connection to the Superalgebras of Gao-Rui-Song-Su}

In this subsection we explain how to recover the affine and cyclotomic walled BrauerClifford superalgebras from [GRSS1] from our supercategories. The discussion here parallels the analogous one in [BCNR, Section 5.5].

First, $\mathcal{A O B} \mathcal{B}$ can be viewed as a $\mathbb{k}\left[\Delta_{1}, \Delta_{3}, \ldots\right]$-linear supercategory with the action of each $\Delta_{k}$ given by tensoring on the right: $h \Delta_{k}:=h \otimes \Delta_{k}$. Given $\delta_{1}, \delta_{2}, \cdots \in \mathbb{k}$, we 
let $\mathcal{A O B} \mathcal{B}\left(\delta_{1}, \delta_{2}, \ldots\right)$ denote the supercategory obtained by specializing each $\Delta_{2 k-1}$ at $\delta_{k}$. In other words, $\mathcal{A O B C}\left(\delta_{1}, \delta_{2}, \ldots\right):=\mathcal{A O B C} \otimes_{\mathbb{k}\left[\Delta_{1}, \Delta_{3}, \ldots\right]} \mathbb{k}$, viewing $\mathbb{k}$ as a $\mathbb{k}\left[\Delta_{1}, \Delta_{3}, \ldots\right]$-module with $\Delta_{2 k-1}$ acting as $\delta_{k}$. It follows from Theorem 3.9 that the superspace $\operatorname{Hom}_{\mathcal{A O B} \mathcal{B}\left(\delta_{1}, \delta_{2}, \ldots\right)}(\mathrm{a}, \mathrm{b})$ has basis consisting of all equivalence classes of normally ordered dotted oriented Brauer-Clifford diagrams (without bubbles) of type $\mathrm{a} \rightarrow \mathrm{b}$. We have a similar specialization for level $\ell$ cyclotomic quotients. Namely, let us write $f(t)=\sum_{i=0}^{\ell} a_{i} t^{i}$. Now, fix $\delta_{1}, \ldots, \delta_{\lfloor\ell / 2\rfloor} \in \mathbb{k}$, and define $\delta_{k}$ recursively for $k>\lfloor\ell / 2\rfloor$ by

$$
\delta_{k}=-\sum_{1 \leq j \leq\lfloor\ell / 2\rfloor} a_{\ell-2 j} \delta_{k-j} .
$$

Then the specialization $\mathcal{O} \mathcal{B C}^{f}\left(\delta_{1}, \ldots, \delta_{[\ell / 2]}\right):=\mathcal{O} \mathcal{B} \mathcal{C}^{f} \otimes_{\mathbb{k}}\left[\Delta_{1}, \Delta_{3}, \ldots\right] \mathbb{k}$. is well defined. By Theorem 7.1, the superspace $\operatorname{Hom}_{\mathcal{O B C}^{f}\left(\delta_{1}, \ldots, \delta_{[\ell / 2]}\right)}(\mathrm{a}, \mathrm{b})$ has basis consisting of all equivalence classes of normally ordered dotted oriented Brauer-Clifford diagrams (without bubbles) of type $\mathrm{a} \rightarrow \mathrm{b}$ with fewer than $\ell \bullet$ 's on each strand. For the remainder of this section we will write $A B C_{s, r}\left(\delta_{1}, \delta_{2}, \ldots\right)$ and $B C_{s, r}^{f}\left(\delta_{1}, \ldots, \delta_{\lfloor\ell / 2\rfloor}\right)$ for the endomorphism algebras of the object $\downarrow^{s} \uparrow^{r}$ in the supercategories $\mathcal{A O B} \mathcal{B}\left(\delta_{1}, \delta_{2}, \ldots\right)$ and $\mathcal{O B C}^{f}\left(\delta_{1}, \ldots, \delta_{\lfloor\ell / 2\rfloor}\right)$, respectively.

Let $B C_{r, s}^{\text {aff }}$ denote the affine walled Brauer-Clifford superalgebra defined in [GRSS1, Definition 3.1]. This superalgebra is defined via odd generators $c_{i}(1 \leq i \leq r), \bar{c}_{j}$ $(1 \leq j \leq s)$; even generators $e_{1}, x_{1}, \bar{x}_{1}, s_{i}(1 \leq i<r), \bar{s}_{j}(1 \leq j<s)$; and even central generators $\omega_{2 k+1}, \bar{\omega}_{k}\left(k \in \mathbb{Z}_{>0}\right)$ subject to a long list of relations. It is an exercise in checking those relations to see that there is a well-defined superalgebra map $B C_{r, s}^{\text {aff }} \rightarrow A B C_{s, r}\left(\delta_{1}, \delta_{2}, \ldots\right)$ defined by

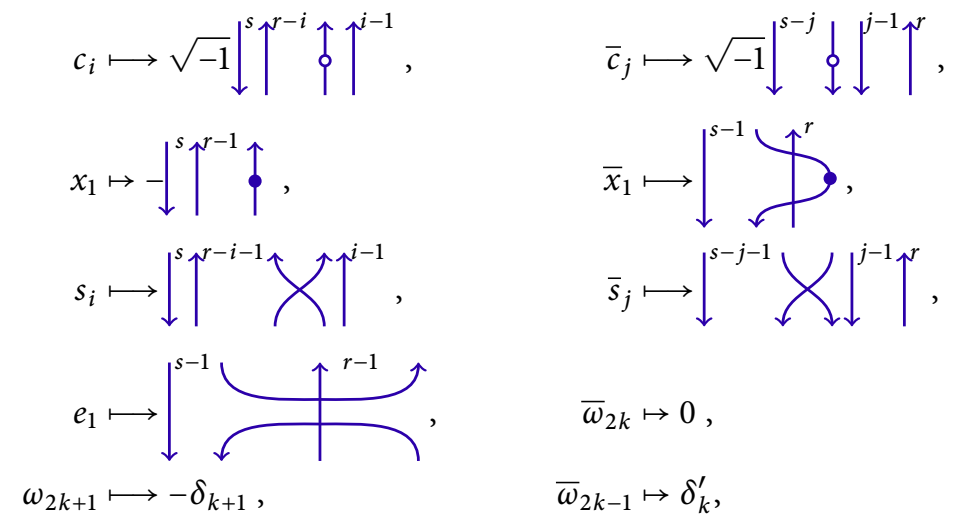

where $\delta_{k}^{\prime}$ is defined recursively by $\delta_{k}-\delta_{k}^{\prime}=\sum_{0<i<\lfloor k / 2\rfloor} \delta_{i} \delta_{k-i}^{\prime}$ (compare with Remark 3.11). This map factors through the quotient $\widetilde{B C}_{r, s}$ of $B C_{r, s}^{\text {aff }}$ by the additional relations $\omega_{2 k-1}=-\delta_{k}, \bar{\omega}_{2 k}=0, \bar{\omega}_{2 k-1}=\delta_{k}^{\prime}$ for all $k \in \mathbb{Z}_{>0}$, which is precisely the specialized superalgebra in [GRSS1, Theorem 5.15]. Using our basis theorem, one can easily check that the spanning set for $\widetilde{B C}_{r, s}$ described in [GRSS1, Definition 3.15 and Corollary 3.16] maps to a basis for $A B C_{s, r}\left(\delta_{1}, \delta_{2}, \ldots\right)$. Hence, $\widetilde{B C}_{r, s} \cong A B C_{s, r}\left(\delta_{1}, \delta_{2}, \ldots\right)$. Note that this also gives a different proof of the linear independence in [GRSS1, Theorem 5.15]. 
A similar discussion applies to cyclotomic quotients. Again, fix $\delta_{1}, \ldots, \delta_{\lfloor\ell / 2\rfloor} \in \mathbb{k}$, and define $\delta_{k}$ for $k>\lfloor\ell / 2\rfloor$ by (7.1). In [GRSS1, Definition 3.14], the cyclotomic walled Brauer-Clifford superalgebra $B C_{\ell, r, s}$ is defined as the quotient of $\widetilde{B C}_{r, s}$ by the additional relations $f\left(x_{1}\right)=g\left(\bar{x}_{1}\right)=0$ where $g(t)$ is another monic degree $\ell$ polynomial satisfying certain conditions. One can check that those conditions imply $f\left(x_{1}\right)$ and $g\left(\bar{x}_{1}\right)$ are mapped to zero under the composition of the isomorphism $B C_{r, s}^{\text {aff }} \rightarrow A B C_{s, r}\left(\delta_{1}, \delta_{2}, \ldots\right)$ from the previous paragraph with the quotient map $A B C_{s, r}\left(\delta_{1}, \delta_{2}, \ldots\right) \rightarrow B C_{s, r}^{f}\left(\delta_{1}, \ldots, \delta_{\lfloor\ell / 2\rfloor}\right)$. Hence, that composition factors through $B C_{\ell, r, s}$ to induce a surjection $B C_{\ell, r, s} \rightarrow B C_{s, r}^{f}\left(\delta_{1}, \ldots, \delta_{\lfloor\ell / 2]}\right)$. Now, using our basis theorem, one can check that the spanning set for $B C_{\ell, r, s}$ from [GRSS1, Definition 3.15 and Corollary 3.16] maps to a basis for $B C_{s, r}^{f}\left(\delta_{1}, \ldots \delta_{\lfloor\ell / 2\rfloor}\right)$. Hence, $B C_{\ell, r, s} \cong B C_{s, r}^{f}\left(\delta_{1}, \ldots, \delta_{\lfloor\ell / 2\rfloor}\right)$.

\section{The Cyclotomic Basis Theorem}

In this section we provide a proof of Theorem 7.1. We actually consider some slightly more general cyclotomic quotients than the ones defined so far, whose definition is similar in spirit to Rouquier's deformed cyclotomic quotients of quiver Hecke algebras from [Rou, $\$ 4.4$ ]. Let $\mathbb{K}$ be some commutative $\mathbb{k}$-algebra and consider the basechanged $\mathbb{K}$-linear monoidal supercategory $\mathcal{A O B} \mathcal{C}_{\mathbb{K}}:=\mathcal{A} \mathcal{O B C} \otimes_{\mathbb{k}} \mathbb{K}$.

Fix $\ell \geq 0$ and monic polynomials

$$
\begin{aligned}
f(u) & =z_{0} u^{\ell}+z_{1} u^{\ell-2}+z_{2} u^{\ell-4}+\cdots, \\
f^{\prime}(u) & =z_{0}^{\prime} u^{\ell}+z_{1}^{\prime} u^{\ell-2}+z_{2}^{\prime} u^{\ell-4}+\cdots
\end{aligned}
$$

in $\mathbb{K}[u]$. So $z_{0}=z_{0}^{\prime}=1$, and all powers of $u$ in these polynomials are even or odd according to the parity of $\ell$. Define the power series

$$
\begin{gathered}
\delta(u)=\delta_{0}+\delta_{1} u^{-1}+\delta_{2} u^{-2}+\cdots, \\
\delta^{\prime}(u)=\delta_{0}^{\prime}+\delta_{1}^{\prime} u^{-1}+\delta_{2}^{\prime} u^{-2}+\cdots
\end{gathered}
$$

in $\mathbb{K}\left[\left[u^{-1}\right]\right]$ from

$$
\begin{aligned}
\delta\left(u^{2}\right) & :=f^{\prime}(u) / f(u), \\
\delta^{\prime}\left(u^{2}\right) & :=-f(u) / f^{\prime}(u) .
\end{aligned}
$$

Note that $\delta_{0}=1$, but $\delta_{0}^{\prime}=-1$. Computing the coefficients of $u^{\ell-2 r}$ in $f^{\prime}(u)=$ $f(u) \delta\left(u^{2}\right)$ gives

$$
\begin{aligned}
& \sum_{s=0}^{r} z_{s} \delta_{r-s}=z_{r}^{\prime} \text { for } r=0, \ldots,\lfloor\ell / 2\rfloor, \\
& \sum_{s=0}^{\lfloor\ell / 2\rfloor} z_{s} \delta_{r-s}=0 \text { for } r>\lfloor\ell / 2\rfloor .
\end{aligned}
$$

Let Sym be the algebra of symmetric functions over $\mathbb{K}$, viewed as a purely even superalgebra. Denote the elementary and complete symmetric functions by $e_{r}$ and $h_{r}$ 
as usual; in particular, $e_{0}=h_{0}=1$. Working in Sym $\left[\left[u^{-1}\right]\right]$, we set

$$
\begin{aligned}
& e(u):=e_{0}+u^{-1} e_{1}+u^{-2} e_{2}+\cdots, \\
& h(u):=h_{0}+u^{-1} h_{1}+u^{-2} h_{2}+\cdots,
\end{aligned}
$$

and recall the fundamental identity $e(u) h(-u)=1$. By Theorem 3.9 and Remark 3.11, there is a well-defined superalgebra isomorphism

(8.6) $\beta: \operatorname{Sym} \stackrel{\sim}{\longrightarrow} \operatorname{End}_{\mathcal{A O B C} \mathcal{E}_{\mathbb{K}}}(\mathbb{1}), \quad h_{r} \longmapsto(-1)^{r} \bigcup_{2 r-1}, \quad e_{r} \longmapsto-2 r-1 \circlearrowright$.

So that this also makes sense in the case $r=0$, it is natural to adopt the convention that $\bigcirc-1:=1_{\mathbb{1}}$ and $-1 \bigcup:=-1_{\mathbb{1}}$.

Lemma 8.1 The $\mathbb{K}$-linear left tensor ideal $\mathcal{J}_{f, f^{\prime}}$ of $\mathcal{A O B} \mathcal{B}_{\mathbb{K}}$ generated by

$$
\left\{f(\hat{\phi}), \bigcirc 2 r-1-\delta_{r} 1_{\mathbb{1}} \mid r=1, \ldots,\lfloor\ell / 2\rfloor\right\}
$$

is generated equivalently by

$$
\left\{f^{\prime}(\downarrow), 2 r-1 \circlearrowright-\delta_{r}^{\prime} 1_{\mathbb{1}} \mid r=1, \ldots,\lfloor\ell / 2\rfloor\right\} .
$$

Moreover, $\mathcal{J}_{f, f^{\prime}}$ contains $\bigcirc{ }^{2 r-1}-\delta_{r} 1_{\mathbb{1}}$ and $2 r-1 \bigcirc-\delta_{r}^{\prime} 1_{1}$ for all $r \geq 0$.

Proof This is similar to [Bru2, Lemma 1.8]. We first show by induction on $r$ that $\mathcal{J}_{f, f^{\prime}}$ contains ${ }_{2 r-1}-\delta_{r} 1_{\mathbb{1}}$ for all $r \geq 0$. This is immediate from the definitions for $r \leq\lfloor\ell / 2\rfloor$, so assume that $r>\lfloor\ell / 2\rfloor$. Since $2 r-1 \geq \ell$, we get from $f(\hat{\varphi}) \in \mathcal{J}_{f, f^{\prime}}$ that $\sum_{s=0}^{\lfloor\ell / 2\rfloor} z_{s} \bigcirc 2(r-s)-1 \in \mathcal{J}_{f, f^{\prime}}$ as well. Now the following verifies the induction step:

$\left.\bigcup_{2 r-1}-\delta_{r} 1_{\mathbb{1}} \stackrel{(8.5)}{=} \mathcal{O}_{2 r-1}+\sum_{s=1}^{\lfloor\ell / 2\rfloor} z_{s} \delta_{r-s} 1_{\mathbb{1}} \equiv \sum_{s=0}^{\lfloor\ell / 2\rfloor} z_{s}\right\}_{2(r-s)-1} \equiv 0 \quad\left(\bmod \mathcal{J}_{f, f^{\prime}}\right)$.

Hence, recalling (8.6), we have that $\beta\left(h\left(-u^{2}\right)\right) \equiv \delta\left(u^{2}\right) 1_{\mathbb{I}}\left(\bmod \mathcal{J}_{f, f^{\prime}}\right)$. Since $e(u)=$ $h(-u)^{-1}$ and $\delta^{\prime}(u)=-\delta(u)^{-1}$, it follows that $\beta\left(e\left(u^{2}\right)\right) \equiv-\delta^{\prime}\left(u^{2}\right) 1_{\mathbb{1}}\left(\bmod \mathcal{J}_{f, f^{\prime}}\right)$. This shows that $2 r-1 \bigcup-\delta_{r}^{\prime} 1_{\mathbb{1}} \in \mathcal{J}_{f, f^{\prime}}$ for all $r \geq 0$. Now we can show that $f^{\prime}(\downarrow) \epsilon$ $\mathcal{J}_{f, f^{\prime}}$ :

$$
\begin{aligned}
f^{\prime}(\downarrow) & =\sum_{r=0}^{\lfloor\ell / 2\rfloor} z_{r}^{\prime} \downarrow \ell-2 r \stackrel{(8.4)}{=} \sum_{r=0}^{\lfloor\ell / 2\rfloor} \sum_{s=0}^{r} z_{s} \delta_{r-s} \downarrow \ell-2 r \equiv \sum_{s=0}^{\lfloor\ell / 2\rfloor} z_{s} \sum_{r=s}^{\lfloor\ell / 2\rfloor} \downarrow \ell-2 r \bigcirc 2(r-s)-1 \\
& =\sum_{s=0}^{\lfloor\ell / 2\rfloor} z_{s} \downarrow{ }_{\ell-2 s} \equiv 0 \quad\left(\bmod \mathcal{J}_{f, f^{\prime}}\right),
\end{aligned}
$$

where for the last equality we have used Proposition 3.10 repeatedly to pull the $\ell-2 s$ dots on the right curl down past the crossing, plus Proposition 3.12 to see many of the dotted bubbles produced are zero. Now we have shown that the left tensor ideal generated by (8.7) contains (8.8). A similar argument shows that the left tensor ideal generated by (8.8) contains (8.7), completing the proof of the lemma. 
Definition 8.2 Define the cyclotomic oriented Brauer-Clifford supercategory associated with the polynomials $f$ and $f^{\prime}$ fixed above to be the $\mathbb{K}$-linear supercategory $\mathcal{O B} \mathcal{C}^{f, f^{\prime}}$ that is the quotient of $\mathcal{A O B} \mathcal{C}_{\mathbb{K}}$ by the $\mathbb{K}$-linear left tensor ideal $\mathcal{J}_{f, f^{\prime}}$ from Lemma 8.1.

Our goal is to establish a basis theorem for the morphism spaces in $\mathcal{O B} \mathcal{C}^{f, f^{\prime}}$. As we will explain fully later on, the cyclotomic oriented Brauer-Clifford supercategory $\mathcal{O B} \mathcal{C}^{f}$ is a special case, so that Theorem 7.1 will follow from this more general result.

Continuing to work over $\mathbb{K}$, recall from Corollary 6.5 that there is a $\mathbb{K}$-superalgebra homomorphism

$$
\alpha: \operatorname{ASerg}_{n} \longrightarrow \operatorname{End}_{\mathcal{A O B} \mathcal{C}_{\mathbb{K}}}\left(\uparrow^{\otimes n}\right)
$$

sending $x_{i}$ to the $\bullet$ on the $i$-th strand, $c_{i}$ to the $\circ$ on the $i$-th strand, and $s_{i}$ to the crossing of the $i$-th and $(i+1)$-th strands (numbering strands $1, \ldots, n$ from right to left). By Theorem 3.9, the map

$$
\alpha \otimes \beta: \operatorname{ASerg}_{n} \otimes_{\mathbb{K}} \operatorname{Sym} \longrightarrow \operatorname{End}_{\mathcal{A O B} \mathcal{C}_{\mathbb{K}}}\left(\uparrow^{\otimes n}\right)
$$

is a superalgebra isomorphism. Let $\operatorname{Serg}_{n}^{f}$ be the cyclotomic Sergeev superalgebra from [BK1, Section 3-e], namely, the quotient of $\mathrm{ASerg}_{n}$ by the two-sided ideal generated

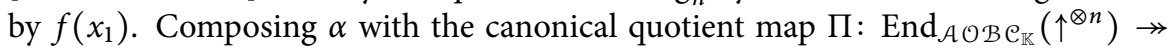

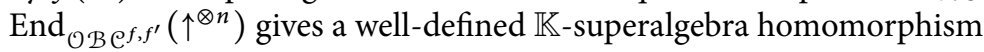

$$
\gamma: \operatorname{Serg}_{n}^{f} \longrightarrow \operatorname{End}_{\mathcal{O B} \mathcal{C}^{f, f^{\prime}}}\left(\uparrow^{\otimes n}\right) .
$$

The following is the key to all our subsequent arguments.

Lemma $8.3 \quad \gamma$ is an isomorphism.

Proof Let $\pi: \operatorname{ASerg}_{n} \otimes_{\mathbb{K}} \operatorname{Sym} \rightarrow \operatorname{Serg}_{n}^{f}$ be the $\mathbb{K}$-superalgebra homomorphism that sends $a \otimes 1$ to the canonical image of $a$ in $\operatorname{Serg}_{n}^{f}$, and $1 \otimes h_{r}$ to $(-1)^{r} \delta_{r}$. Note that $\operatorname{ker} \pi$ is $I \otimes \operatorname{Sym}+\operatorname{ASerg}_{n} \otimes J$, where $I$ is the two-sided ideal of ASerg ${ }_{n}$ generated by $f\left(x_{1}\right)$ and $J$ is the two-sided ideal of Sym generated by $h_{r}-(-1)^{r} \delta_{r}$ for $r \geq 1$. Also let $\Pi: \operatorname{End}_{\mathcal{A O B} \mathcal{C}_{\mathrm{K}}}\left(\uparrow^{\otimes n}\right) \rightarrow \operatorname{End}_{\mathcal{O B} \mathcal{C}^{f, f^{\prime}}}\left(\uparrow^{\otimes n}\right)$ be the canonical quotient map as above. By directly checking it on generators, one sees that the following diagram commutes:

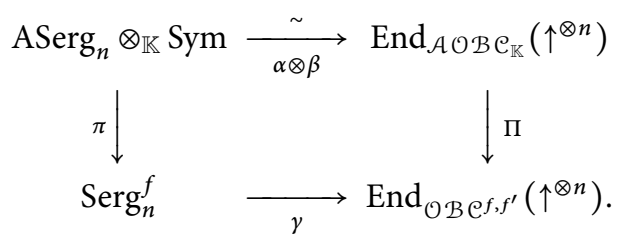

It follows immediately that $\gamma$ is surjective. Moreover, the injectivity of $\gamma$ follows if we can show that $(\alpha \otimes \beta)^{-1}(\operatorname{ker} \Pi) \subseteq \operatorname{ker} \pi$. 
By the definition of $\mathcal{O B} \mathcal{C}^{f, f^{\prime}}, \operatorname{ker} \Pi$ is the subspace of $\operatorname{End}_{\mathcal{A O B}} \mathcal{C}_{\mathbb{K}}\left(\uparrow^{\otimes n}\right)$ defined by the left tensor ideal $\mathcal{J}_{f, f^{\prime}}$. This means that any element of $\operatorname{ker} \Pi$ is a $\mathbb{K}$-linear combination of morphisms $\theta: \uparrow^{\otimes n} \rightarrow \uparrow^{\otimes n}$ in $\mathcal{A O B} \mathcal{C}_{\mathbb{K}}$ of the form

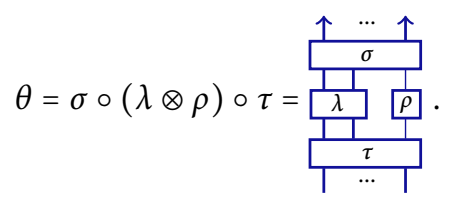

where $\rho$ is one of the generating morphisms $f(\hat{\phi})$ or $\beta\left(h_{r}\right)-(-1)^{r} \delta_{r}$ for $\mathcal{J}_{f, f^{\prime}}$, and $\sigma, \tau, \lambda$ are any other morphisms so that the compositions make sense. Thus, we must show that the inverse image under $\alpha \otimes \beta$ of such a morphism $\theta=\sigma \circ(\lambda \otimes \rho) \circ \tau$ lies in $\operatorname{ker} \pi=I \otimes \operatorname{Sym}^{2} \operatorname{ASerg}_{n} \otimes J$.

If $\rho=\beta\left(h_{r}\right)-(-1)^{r} \delta_{r}$ for some $r$, then $(\alpha \otimes \beta)^{-1}(\theta)$ obviously lies in $\mathrm{ASerg}_{n} \otimes J$. Instead, suppose that $\rho=f(\hat{\phi})$. Using the relations established earlier in the paper (especially Propositions 3.10 and 3.12), we "straighten" the diagram $\theta$ leaving the $\rho$ coupon on the right edge fixed, to rewrite it as a $\mathbb{K}$-linear combination of morphisms of the following two types:

(I)

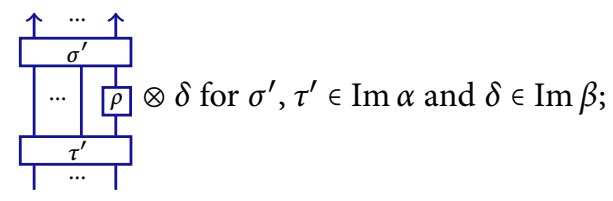

(II) $\left.\begin{array}{ll}\uparrow & \cdots \\ \lambda^{\prime} & \cdots\end{array}\right\} \bigodot^{2 r-\ell-1} \otimes \delta$ for $\lambda^{\prime} \in \operatorname{Im}(\alpha), \delta \in \operatorname{Im}(\beta)$ and $r>\lfloor\ell / 2\rfloor$.

These morphisms arise when the $\rho$-coupon ends up on a propagating strand (type I) or on a dotted bubble (type II) after straightening. The inverse image under $\alpha \otimes \beta$ of a type I morphism lies in $I \otimes$ Sym. The inverse image under $\alpha \otimes \beta$ of a type II morphism lies in $\mathrm{ASerg}_{n} \otimes J$ because

$$
\begin{aligned}
\beta^{-1}\left(\bigodot_{\rho}^{2 r-\ell-1}\right) & =\sum_{s=0}^{\lfloor\ell / 2\rfloor}(-1)^{r-s} z_{s} h_{r-s} \\
& \stackrel{(8.5)}{=} \sum_{s=0}^{\lfloor\ell / 2\rfloor}(-1)^{r-s} z_{s}\left(h_{r-s}-(-1)^{r-s} \delta_{r-s}\right) \in J .
\end{aligned}
$$

Now we can prove the main result of this section. Recall the definition of normally ordered dotted oriented Brauer-Clifford diagram from Definition 3.8.

Theorem 8.4 For any $a, b \in \mathrm{ob} \mathcal{O B} \mathcal{C}^{f, f^{\prime}}$, the morphism space $\operatorname{Hom}_{\mathcal{O B}} \mathcal{C}_{f, f^{\prime}}(a, b)$ is a free $\mathbb{K}$-module with basis given by equivalence classes of normally ordered dotted oriented Brauer-Clifford diagrams (without bubbles) of type $a \rightarrow b$ having fewer than $\ell$ -'s on each strand.

Proof Let us first show that the given diagrams span $\operatorname{Hom}_{\mathcal{O B} \mathcal{C}^{f, f^{\prime}}}(\mathrm{a}, \mathrm{b})$. In fact, we show by induction on $N$ that any diagram representing a morphism $\mathrm{a} \rightarrow \mathrm{b}$ in 
$\mathcal{O B} \mathcal{C}^{f, f^{\prime}}$ with $N$ 's can be written as a linear combination of the ones among the specified diagrams that have $N$ or fewer $\bullet$. The case $N=0$ is straightforward (and also follows from Theorem 3.4). In general, take a diagram with $N>0 \bullet$ 's. Using the relations, $\bullet$ 's and o's can be moved past crossings or other dots (possibly introducing a sign in the case of o's) modulo diagrams with strictly fewer $\bullet$ 's. In particular, any dotted bubble can be moved to the right-hand side of the picture modulo diagrams with strictly fewer 's and, once on the right-hand side, it may be replaced by a scalar using Proposition 3.12 and the last part of Lemma 8.1. To complete the proof of the spanning part of the theorem, it remains to observe that if any strand has $\ell \bullet$ 's, it can be rewritten in terms of diagrams with strictly fewer $\bullet$ 's: for any objects a and $b$, the relations $\mathrm{b} \otimes \mathrm{a} \otimes f(\hat{\$})=0$ and $\mathrm{b} \otimes \mathrm{a} \otimes f^{\prime}(\downarrow)=0$ in $\mathcal{O} \mathcal{B} \mathcal{C}^{f, f^{\prime}}$ imply that

$$
\left.\left.\right|_{\mathrm{b}} \uparrow_{e} \equiv\right|_{\mathrm{a}} \bigoplus_{\mathrm{b}} e \equiv 0,\left.\left.\quad \oint_{\mathrm{a}}\right|_{\mathrm{b}} \equiv\right|_{\mathrm{a}} \bigoplus_{\mathrm{b}} e \equiv 0,
$$

where $\equiv$ means "equal modulo a linear combination of diagrams with fewer than $\ell \bullet$ 's."

It remains to establish linear independence. Note to start with that the result is true in the special case $\mathrm{a}=\mathrm{b}=\uparrow^{\otimes n}$, for in this case it follows using Lemma 8.3 and the basis theorem for $\operatorname{Serg}_{n}^{f}$ established in [BK1, $\$ 3$-e]. In general, we first reduce to the case $\mathrm{b}=\mathbb{1}$ using $\mathbb{K}$-module isomorphism

$$
\operatorname{Hom}_{\mathcal{O B} \mathcal{C}^{f, f^{\prime}}}(\mathrm{a}, \mathrm{b}) \stackrel{\sim}{\longrightarrow} \operatorname{Hom}_{\mathcal{O B} \mathcal{B}^{f, f^{\prime}}}\left(\mathrm{b}^{*} \otimes \mathrm{a}, \mathbb{1}\right), \quad
$$

This same reduction proves the theorem in the case $\mathrm{a}=\downarrow^{\otimes n} \otimes \uparrow^{\otimes n}, \mathrm{~b}=\mathbb{1}$, since it follows from the special case $\mathrm{a}=\mathrm{b}=\uparrow^{\otimes n}$ treated already.

Now suppose that $a$ is arbitrary and $b=\mathbb{1}$. The space $\operatorname{Hom}_{\mathcal{O B} \mathcal{C}^{f, f^{\prime}}}(\mathrm{a}, \mathbb{1})$ is zero unless a has $n$ letters equal to $\uparrow$ and $n$ letters equal to $\downarrow$ for some $n \geq 0$. Assuming that is the case, the object a is a "shuffle" of the tensor $\downarrow^{\otimes n} \otimes \uparrow \otimes n$ already treated. Consider the minimal length permutation of tensor factors taking a to $\downarrow^{\otimes n} \otimes \uparrow \otimes n$. There is corresponding isomorphism $w: \mathrm{a} \stackrel{\sim}{\rightarrow} \downarrow^{\otimes n} \otimes \uparrow^{\otimes n}$ in $\mathcal{O B} \mathcal{C}^{f, f^{\prime}}$ obtained by composing various rightward crossings. Hence, we get another $\mathbb{K}$-module isomorphism

$$
\operatorname{Hom}_{\mathcal{O} \mathcal{B} \mathcal{C}^{f, f^{\prime}}}\left(\downarrow^{\otimes n} \otimes \uparrow \uparrow^{\otimes n}, \mathbb{1}\right) \stackrel{\sim}{\longrightarrow} \operatorname{Hom}_{\mathcal{O B} \mathcal{B} \mathcal{C}^{f, f^{\prime}}}(\mathrm{a}, \mathbb{1}), \quad \theta \longmapsto w \circ \theta .
$$

Applying this isomorphism to the basis for $\operatorname{Hom}_{\mathcal{O B C} \mathcal{C}^{f, f^{\prime}}}\left(\downarrow^{\otimes n} \otimes \uparrow^{\otimes n}, \mathbb{1}\right)$ already obtained at the end of the previous paragraph, we obtain a basis for $\operatorname{Hom}_{\mathcal{O B} \mathcal{C}^{f, f^{\prime}}}(\mathrm{a}, \mathbb{1})$. It is not quite the same as the basis of normally ordered diagrams we are after, but from it we can slide 's and o's along strands to obtain the desired basis (up to some signs), modulo diagrams with strictly fewer 's, just like in the opening paragraph of this proof. This means that the transition matrix between the basis in hand and the basis in mind is unitriangular when suitably ordered, which is all that is needed to complete the proof.

Finally, we explain the connection to the cyclotomic quotients $\mathcal{O B} \mathcal{C}^{f}$. For this, we specialize to the case that the monic polynomial $f(u)$ from (8.1) has its coefficients in 

the original ground field $\mathbb{k}$. Also let

$$
f^{\prime}(u):=u^{\ell}+z_{1}^{\prime} u^{\ell-2}+\cdots \in \mathbb{K}[u],
$$

where $\mathbb{K}:=\mathbb{k}\left[z_{1}^{\prime}, \ldots, z_{\lfloor\ell / 2\rfloor}^{\prime}\right]$ for indeterminates $z_{1}^{\prime}, \ldots, z_{\lfloor\ell / 2\rfloor}^{\prime}$. Define $\delta(u), \delta^{\prime}(u)$ from (8.2) and (8.3). By the identity (8.4), the coefficients $\delta_{1}, \ldots, \delta_{\lfloor\ell / 2\rfloor}$ of $\delta(u)$ are related to the indeterminates $z_{1}^{\prime}, \ldots, z_{\lfloor\ell / 2\rfloor}^{\prime}$ by a unitriangular transition matrix. Thus, $\mathbb{K}$ is also freely generated by $\delta_{1}, \ldots, \delta_{\lfloor\ell / 2\rfloor}$. In $\mathcal{O B} \mathcal{C}^{f, f^{\prime}}$, the scalar $\delta_{r}$ acts in the same way as tensoring on the right with the counterclockwise bubble with $2 r-1 \bullet$ 's. So we get the following corollary immediately from Theorem 8.4.

Corollary 8.5 Assume $f(u) \in \mathbb{k}[u]$ and $f^{\prime}(u) \in \mathbb{K}[u]$ as in (8.9). For objects $a, b$ in $\mathcal{O B} \mathcal{C}^{f, f^{\prime}}$, the morphism space $\operatorname{Hom}_{\mathcal{O B}} \mathcal{C}^{f, f^{\prime}}(a, b)$ has basis as a vector space over $\mathbb{k}$ given by equivalence classes of normally ordered dotted oriented Brauer-Clifford diagrams with bubbles of type $a \rightarrow b$ having fewer than $\ell \bullet$ 's on each strand.

Continuing with $f(u) \in \mathbb{k}[u]$, the $\mathbb{k}$-linear supercategory $\mathcal{O B} \mathcal{C}^{f}$ is the quotient of $\mathcal{A O B C}$ by the $\mathbb{k}$-linear left tensor ideal generated by $f(\hat{\phi})$. The composition of the natural $\mathbb{k}$-linear superfunctor $\mathcal{A O B C} \rightarrow \mathcal{A O B C} \mathcal{C}_{\mathbb{K}}$ followed by the quotient functor $\mathcal{A O B C} \mathcal{C}_{\mathbb{K}} \rightarrow \mathcal{O B} \mathcal{C}^{f, f^{\prime}}$ factors through $\mathcal{O B} \mathcal{C}^{f}$ to induce a $\mathbb{k}$-linear superfunctor

$$
\mathcal{O B C} \mathrm{C}^{f} \longrightarrow \mathcal{O B C}^{f, f^{\prime}} \text {. }
$$

This is the identity on objects. On morphisms, it is noted already in Section 7.1 that $\operatorname{Hom}_{\mathcal{O B} \mathcal{C}^{f}}(\mathrm{a}, \mathrm{b})$ is spanned as a vector space over $\mathbb{k}$ by the equivalence classes of normally ordered dotted oriented Brauer-Clifford diagrams with bubbles of type $a \rightarrow b$ having fewer than $\ell \bullet$ 's on each strand; the proof is the same argument as in the first paragraph of the proof of Theorem 8.4. The images of these spanning morphisms under the superfunctor $(8.10)$ give a basis for $\operatorname{Hom}_{\mathcal{O B} \mathcal{C}^{f, f^{\prime}}}(\mathrm{a}, \mathrm{b})$ thanks to Corollary 8.5. Hence, they are already linearly independent in $\operatorname{Hom}_{\mathcal{O B}^{f}}(\mathrm{a}, \mathrm{b})$, and (8.10) is an isomorphism on morphism spaces. We have proved the following corollary.

Corollary 8.6 The $\mathbb{k}$-linear superfunctor (8.10) is an isomorphism.

Corollaries 8.5 and 8.6 together imply Theorem 7.1.

Acknowledgments The authors are pleased to thank Nicholas Davidson and Catharina Stroppel for helpful conversations. Part of this project was completed while the second author enjoyed a visit to the Max Planck Institute in Bonn. He would like to thank the institute for providing an excellent working environment.

\section{References}

[BDE+] M. Balagovic, Z. Daugherty, I. Entova-Aizenbud, I. Halacheva, J. Hennig, M. S. Im, G. Letzter, E. Norton, V. Serganova, and C. Stroppel, The affine VW supercategory. 2018. arxiv: 1801.04178

[BGH+] G. Benkart, N. Guay, J. H. Jung, S.-J. Kang, and S. Wilcox, Quantum walled Brauer-Clifford superalgebras. J. Algebra 454(2016), 433-474. http://dx.doi.org/10.1016/j.jalgebra.2015.04.038

[BD1] D. Benson and S. Doty, Schur-Weyl duality over finite fields. Arch. Math. (Basel) 93(2009), 425-435. http://dx.doi.org/10.1007/s00013-009-0066-8 
[Brul] J. Brundan, Kazhdan-Lusztig polynomials and character formulae for the Lie superalgebra $\mathfrak{q}(n)$. Adv. Math. 182(2004), 28-77. http://dx.doi.org/10.1016/S0001-8708(03)00073-2

[Bru2] — On the definition of Heisenberg category. Algebr. Comb. 1(2018), 523-544. http://dx.doi.org/10.5802/alco.26

[Bru3] $\longrightarrow$ Representations of oriented skein categories. 2017. arxiv:1712.08953

[BCNR] J. Brundan, J. Comes, D. Nash, and A. Reynolds, A basis theorem for the affine oriented Brauer category and its cyclotomic quotients. Quantum Topol. 8(2017), 75-112. http://dx.doi.org/10.4171/QT/87

[BD2] J. Brundan and N. Davidson, Type A blocks of super category $\mathcal{O}$. J. Algebra 473(2017), 447-480. http://dx.doi.org/10.1016/j.jalgebra.2016.11.022

[BD3] — Type C blocks of super category $\mathcal{O}$. 2017. arxiv:1702.05055

[BE1] J. Brundan and A. P. Ellis, Monoidal supercategories. Comm. Math. Phys. 351(2017), 1045-1089, http://dx.doi.org/10.1007/s00220-017-2850-9

[BE2] — Super Kac-Moody 2-categories. Proc. Lond. Math. Soc. 115(2017), no. 5, 925-973. http://dx.doi.org/10.1112/plms.12055

[BK1] J. Brundan and A. Kleshchev, Hecke-Clifford superalgebras, crystals of type $A_{21}^{(2)}$ and modular branching rules for $\widehat{S}_{n}$. Represent. Theory 5(2001), 317-403. http://dx.doi.org/10.1090/S1088-4165-01-00123-6

[BK2] - Projective representations of symmetric groups via Sergeev duality. Math. Z. 239(2002), 27-68. http://dx.doi.org/10.1007/s002090100282

[BK3] — Modular representations of the supergroup Q(n). I. J. Algebra 260(2003), 64-98. http://dx.doi.org/10.1016/S0021-8693(02)00620-8

[Che] C.-W. Chen, Reduction method for representations of queer Lie superalgebras. J. Math. Phys. 57(2016), no. 5, 051703. http://dx.doi.org/10.1063/1.4948744

[CKW] S.-J. Cheng, J.-H. Kwon, and W. Wang, Character formulae for queer Lie superalgebras and canonical bases of types A/C. Comm. Math. Phys. 352(2017), 1091-1119. http://dx.doi.org/10.1007/s00220-016-2809-2

[CW] S.-J. Cheng and W. Wang, Dualities and representations of Lie superalgebras. Graduate Studies in Mathematics, 144, American Mathematical Society, Providence, RI, 2012. http://dx.doi.org/10.1090/gsm/144

[CM] K. Coulembier and V. Mazorchuk, The G-centre and gradable derived equivalences. 2017. arxiv:1703.02623

[DW] J. Du and J. Wan, Presenting queer Schur superalgebras. Int. Math. Res. Not. IMRN 2015, no. 8, 2210-2272. http://dx.doi.org/10.1093/imrn/rnt262

[EL] A. P. Ellis and A. D. Lauda, An odd categorification of $U_{q}\left(\mathfrak{s l}_{2}\right)$. Quantum Topol. 7(2016), 329-433. http://dx.doi.org/10.4171/QT/78

[GRSS1] M. Gao, H. Rui, L. Song, and Y. Su, Affine walled Brauer-Clifford superalgebras. 2017. arxiv:1708.05135

[GRSS2] —. A proof of Comes-Kujawa's conjecture. 2018. arxiv:1801.09071

[HKS] D. Hill, J. R. Kujawa, and J. Sussan, Degenerate affine Hecke-Clifford algebras and type Q Lie superalgebras. Math. Z. 268(2011), 1091-1158. http://dx.doi.org/10.1007/s00209-010-0712-7

[JK] J. Jung and S. Kang, Mixed Schur-Weyl-Sergeev duality for queer Lie superalgebras. J. Algebra 399(2014), 516-545. http://dx.doi.org/10.1016/j.jalgebra.2013.08.029

[KKO1] S.-J. Kang, M. Kashiwara, and S.-j. Oh, Supercategorification of quantum Kac-Moody algebras. Adv. Math. 242(2013), 116-162. http://dx.doi.org/10.1016/j.aim.2013.04.008

[KKO2] — Supercategorification of quantum Kac-Moody algebras II. Adv. Math. 265(2014), 169-240. http://dx.doi.org/10.1016/j.aim.2014.07.036

[Kle] A. Kleshchev, Linear and projective representations of symmetric groups. Cambridge Tracts in Mathematics, 163, Cambridge University Press, Cambridge, 2005. http://dx.doi.org/10.1017/СВО9780511542800

[KT] J. R. Kujawa and B. Tharp, The marked Brauer category. J. Lond. Math. Soci. 95(2017), 393-413. http://dx.doi.org/10.1112/jlms.12015

[Naz] M. Nazarov, Young's symmetrizers for projective representations of the symmetric group. Adv. Math. 127(1997), 190-257. http://dx.doi.org/10.1006/aima.1997.1621

[PS] I. Penkov and V. Serganova, Characters of irreducible G-modules and cohomology of $G / P$ for the Lie supergroup $G=Q(N)$. Algebraic geometry, 7, J. Math. Sci. (New York) 84(1997), 1382-1412. http://dx.doi.org/10.1007/BF02399196

[Rey] A. Reynolds, Representations of the oriented Brauer category. PhD thesis, University of Oregon, Eugene, Oregon, 2015. 


\section{A BasisTheorem for the DegenerateAffine Oriented Brauer-Clifford Supercategory 1101}

[RS] D. Rosso and A. Savage, A general approach to Heisenberg categorification via wreath product algebras. Math. Z. 286(2017), 603-655. http://dx.doi.org/10.1007/s00209-016-1776-9

[Rou] R. Rouquier, Quiver Hecke algebras and 2-Lie algebras. Algebra Colloq. 19(2012), 359-410. http://dx.doi.org/10.1142/S1005386712000247

[Ser1] A. N. Sergeev, The centre of enveloping algebra for Lie superalgebra $Q(n, C)$. Lett. Math. Phys. 7(1983), 177-179. http://dx.doi.org/10.1007/BF00400431

[Ser2] Tensor algebra of the identity representation as a module over the Lie superalgebras $\mathrm{Gl}(n, m)$ and $Q(n)$. Mat. Sb. (N.S.) 123(165), 422-430.

Department of Mathematics, University of Oregon, Eugene, Oregon 97403-1222, USA

Email: brundan@uoregon.edu

Department of Mathematics \& Physical Sciences, The College of Idaho, Caldwell, Idaho 83605, United States

Email: jonnycomes@gmail.com

Department of Mathematics, University of Oklahoma, Norman, Oklahoma 73019-0315, United States Email: kujawa@math.ou.edu 